\title{
Examples of character varieties in characteristic $p$ and ramification
}

\author{
Luisa Paoluzzi and Joan Porti*
}

May 20, 2019

\begin{abstract}
We study $\mathrm{SL}_{2}(\mathbb{F})$-character varieties of knots over algebraically closed fields $\mathbb{F}$. We give a sufficient condition in terms of the double branched cover of a 2-bridge knot (or, equivalently, of its Alexander polynomial) on the characteristic of $\mathbb{F}$, an odd prime, for the $\mathrm{SL}_{2}(\mathbb{F})$-character variety to present ramification phenomena. Finally we provide several explicit computations of character varieties to illustrate the result, exhibiting also other types of ramification.
\end{abstract}

\section{Introduction}

Character varieties have turned out to be powerful tools in the comprehension of 3-manifolds in general and knots in particular. In the case of knots, different invariants are related or connected to character varieties. This is, for instance, the case of the A-polynomial 5, and the algebraic and geometric properties of the excellent component of a hyperbolic knot 10, 15, 22. In this paper we are interested in a far less explored type of invariants, namely the finite set of odd prime numbers $p$ for which the character variety ramifies in characteristic $p$.

Recall that for a finitely presented group $\Gamma$ its $\mathrm{SL}_{2}(\mathbb{C})$-character variety, noted $X(\Gamma)$, is an algebraic set parameterizing, roughly speaking, the conjugacy classes of representations of $\Gamma$ into $\mathrm{SL}_{2}(\mathbb{C})$. The algebraic set $X(\Gamma)$ is determined by a finite set of polynomial equations with integers coefficients. If $p$ is a prime number, one can consider the polynomial equations obtained from the previous ones by reducing their coefficients mod $p$. It follows from work by GonzálezAcuña and Montesinos-Amilibia 8 that these new equations define the variety of characters of representations of $\Gamma$ into $\mathrm{SL}_{2}(\mathbb{F})$, where $\mathbb{F}$ is an algebraically closed field of characteristic $p$, provided that $p$ is odd.

It is not hard to see that for almost every $p$ several features of the structure of the variety of characters of $\Gamma$ over $\mathbb{F}$ coincide with those of $X(\Gamma)$ over $\mathbb{C}$ : this is for instance the case of the number of irreducible components of the algebraic set and their dimensions. We shall say that $p$ ramifies if there is a discrepancy between the behaviour of $X(\Gamma)$ and that of the character variety in characteristic $p$. We are interested in understanding what types of ramification phenomena can appear in character varieties of knots (i.e. when $\Gamma$ is the fundamental group of a knot exterior) and which primes ramify.

\footnotetext{
*Partially supported by the Spanish Mineco through grant MTM2015-66165-P
} 
The existence of two different types of ramification phenomena were pointed out in previous papers by the authors. Both variations in the dimensions of irreducible components and in their number can occur: the former phenomenon appears for character varieties of orbifold structures of certain Montesinos knots [20], and the latter for knots admitting symmetries of order $p$ [21]. In both cases, the appearance of ramification phenomena is related to the elementary fact that a matrix in $\mathrm{SL}_{2}(\mathbb{F})$ has order $p$ if and only if it is parabolic (or unitary), i.e. has trace equal to 2 .

The present paper is devoted to exhibiting examples of yet another type of ramification phenomena that we might describe as order-1 ramification in contrast to the ramification observed so far that we might call of order 0 .

To make this discussion more concrete, let us consider an explicit example: the character variety of the figure-eight knot. It is well-known that this algebraic set can be defined by the equation $(x-2)\left(x^{2}+\left(1-t^{2}\right) x+t^{2}-1\right)$ where $t$ represents the trace of a meridian, while $x$ is the trace of the product of two generators of the group: a meridian and a conjugate of its inverse (see for instance [8, Section 6], with the variables $z=t^{2}-x$ and $\left.y=t^{2}\right)$. The character variety consists of two 1-dimensional irreducible components: the first one is the component of characters of abelian representations, while the second one is Thurston's excellent component containing the character of the lift to $\mathrm{SL}_{2}(\mathbb{C})$ of the hyperbolic holonomy of the knot. It is easy to convince oneself that, regardless of $p$, the variety of $\mathrm{SL}_{2}(\mathbb{F})$ always consists of two 1-dimensional irreducible components. However, although both components are smooth and intersect in precisely two points over $\mathbb{C}$, this is no longer the case for all $p$. Indeed, by computing the partial derivatives of the second component one has $2 x-t^{2}+1$ and $-2 t(x-1)$ and one realises that, for $p=5$, the second component is no more smooth at the point $t=0$ and $x=2$ : the point is a cuspidal point and the two points of intersection between the two components collapse to this singular one in characteristic 5. Observe that if one considers $\mathrm{PSL}_{2}$-characters instead of $\mathrm{SL}_{2}$-characters, that is if we take $T=t^{2}$ as a coordinate instead of $t$, then the point of coordinates $T=0$ and $x=2$ is smooth even in characteristic 5 on each of the components, but the intersection between both components is transverse in characteristic zero or $p \neq 5$, and tangent in characteristic $p=5$.

Definition 1. We say that $X(\Gamma)$ has a ramification of order 1 type in characteristic $p$ if in the character variety in characteristic $p$ either the type of intersection beteen two irreducible components changes (in particular, two transverse components become tangent), or the singularity type of a point changes (in particular, a singular point appears on a smooth component).

Note that ramifications of order 0 and 1 may appear at the same time if, for instance, an irreducible component splits into two and they meet at a point.

The following result gives a conceptual explanation of the reason why 5 is a ramified prime for the character variety of the figure-eight knot.

Theorem 1. Let $K$ be a 2-bridge knot, $\Delta_{K}(t)$ its Alexander polynomial, and $p$ a prime. We consider $X\left(\pi_{1}(K)\right)$. If $p$ divides $\left|\Delta_{K}(-1)\right|$, that is the order of the homology of the 2-fold branched cover of $K$, then $p$ ramifies.

The above is a special case of more technical result for whose statement we need to introduce some notation first. Let $K$ be a knot and $M_{n}$ its $n$-fold cyclic 
branched cover. Let $\mu \in \pi_{1}(K)$ be an element representing a meridian of $K$. We have the following exact sequence of groups

$$
1 \longrightarrow H \longrightarrow \pi_{1}(K) /<<\mu^{4}>>\longrightarrow \mathbb{Z} / 2 \mathbb{Z} \longrightarrow 1 .
$$

where $H$ can be seen as the orbifold fundamental group of the orbifold whose underlying topological space is $M_{2}$ and whose singular locus is the lift of $K$ with order 2 singularity. The commutator subgroup $H^{\prime}$ of $H$ is characteristic, so we can consider the quotient $\Gamma=\left(\pi_{1}(K) /<<\mu^{4}>>\right) / H^{\prime}$.

Theorem 2. Let $K$ be a knot, $\Delta_{K}(t)$ its Alexander polynomial, and $p$ a prime. We consider $X(\Gamma)$, where $\Gamma$ is defined as above. If $p$ divides $\left|\Delta_{K}(-1)\right|$, that is the order of $H_{1}\left(M_{2}\right)$, then $p$ ramifies for $X(\Gamma)$.

Remark that, for 2-bridge knots, $\Gamma$ is a binary dihedral group, extension of $\mathbb{Z} / 2 \mathbb{Z}$ by $\pi_{1}\left(M_{2}\right)=H_{1}\left(M_{2}\right)$. In addition, $\mathrm{SL}_{2}(\mathbb{C})$-representations of $\Gamma$ are lifts of representations of $\pi_{1}(K) /<<\mu^{2}>>$ to $\mathrm{PSL}_{2}(\mathbb{C})$.

A natural question is to determine what ramification phenomena can appear in the situation of Theorem 2 and if the ramification is for $X(K)$ itself and not just for its algebraic subset $X(\Gamma)$. The easiest class of examples to consider is that of torus knots (see Section 7). Over $\mathbb{C}$ the character variety of a torus knot consists of a finite number of rational curves and the only ramification phenomena that occur are when several irreducible components collapse onto a single one. It follows that the character variety of the torus knot $T(a, b)$ ramifies for every odd prime $p$ dividing $a b$, in particular whenever $p$ divides $\left|\Delta_{T(a, b)}(-1)\right|$.

Although torus knots show that the ramification phenomena that can be observed in the situation of Theorem 2 is not always of the type seen for the figure-eight knot, one might expect that this latter behaviour is generic, at least for hyperbolic knots. The following corroborates this hypothesis.

Proposition 3. Let $K$ be a hyperbolic 2-bridge knot with Alexander polynomial $\Delta_{K}(t)$. Let $p$ be a divisor of $\left|\Delta_{K}(-1)\right|$. If all roots of $\Delta_{K}$ are simple, then the ramification phenomena occurring at $p$ is of order 1 .

By [9] the hypothesis that all roots of $\Delta_{K}(t)$ are simple implies that the curve of abelian characters intersects transversely the other components of $X(K)$. In Lemma 9 we show that the intersection is no more transverse but tangent in characteristic $p$ whenever $p$ divides $\left|\Delta_{K}(-1)\right|$. Furthermore, the arguments of [9] can be used to argue that ramifications in the intersection of both components will appear at most for primes that divide the discriminant of $\Delta_{K}(t)$, provided this discriminant does not vanish (namely, all roots of $\Delta_{K}(t)$ are simple). See Remark 2. Notice that $\Delta_{K}(-1)$ divides the discriminant of $\Delta_{K}(t)$.

Incidentally, the techniques of the proof yield the following fact which does not seem to be stressed elsewhere in the literature.

Proposition 4. Let $K$ be a hyperbolic 2-bridge knot with Alexander polynomial $\Delta_{K}(t)$. Let $\alpha=\left|\Delta_{K}(-1)\right|$. If $\alpha$ and $(\alpha-1) / 2$ are prime numbers, then the character variety of $K$ over $\mathbb{C}$ consists precisely of two irreducible components: the one whose characters correspond to abelian representations and Thurston's excellent one.

At this point we do not know whether the condition $(\alpha-1) / 2$ prime in the above proposition is in fact necessary. On the other hand, there are examples of 
hyperbolic 2-bridge knots such that neither $\alpha$ nor $(\alpha-1) / 2$ are prime numbers and yet their character varieties consist of just two irreducible components.

To complete our analysis and to improve our understanding of ramification phenomena, we provide explicit computations of character varieties for some knots, notably for the tunnel number-one $P(2,2,3)$-pretzel knot $8_{5}$ and for the $\pi$-hyperbolic knot $8_{18}$. We also study character varieties for some 2 -bridge knots. Among them, the knot $8_{9}$ admits a ramified prime that does not come from the situation of Theorem 2. Quite interestingly, in this case we observe a new type of ramification, namely an irreducible component splitting into two irreducible ones.

Note that the aforementioned examples cover all possible geometries of double branched covers of hyperbolic knots, that is spherical, Seifert fibred, and hyperbolic. As for the case of hyperbolic knots with double branched covers admitting a non trivial JSJ-decomposition, their character varieties seem hard to compute because of the "large number" of generators of their groups (none of these is a tunnel number-one knot, for instance). Instead, we compute explicitly the character varieties of two tunnel number-one satellite knots, both with companion the trefoil knot. The first is a cable knot whose double branched cover is a graph manifold, while the second is a Whitehead double so the JSJdecomposition of its double branched cover contains a hyperbolic piece. As a side remark, the double branched covers of these two knots are also the double branched covers of hyperbolic knots. Of course, it is not clear if there might be a relationship between the character varieties of two knots sharing the same double cover.

The paper is organised as follows. In section 2 we recall basic facts about character varieties. The proofs of Theorems 1 and 2 follow from the discussion in Section 3, while Section 4 deals with the specificities of 2-bridge knots. This latter section also contains detailed computations of character varieties for some 2 -bridge knots and the primes for which they ramify. The character varieties of the knots $8_{5}$ and $8_{18}$ are studied in Sections 5 and 6 , respectively. Section 7 is dedicted to character varieties of torus knots, while in Section 8 we discuss two examples of character varieties of satellite knots. Finally, some auxiliary computations are provided in the Appendices.

The computations in this paper were performed by using Maple ${ }^{\mathrm{TM}}$.

Acknowledgements The authors are indebted to Alan Reid for suggesting the exploration of order 1 type ramification. L. Paoluzzi is also thankful to $\mathrm{UAB}$ for hospitality during her visit when the contents of the present paper were originally discussed.

\section{Varieties of characters}

In this section we recall relevant facts about character varieties and introduce some notation.

Let $\Gamma$ be a finitely presented group and $\mathbb{F}$ an algebraically closed field. Given a representation $\rho: \Gamma \longrightarrow \mathrm{SL}_{2}(\mathbb{F})$, one can define a map $\chi_{\rho}: \Gamma \longrightarrow \mathbb{F}$ by composing with the trace function. Such map is called the character of the representation $\rho$. We will denote $X(\Gamma)_{\mathbb{F}}$ the set of all characters of representations of 
$\Gamma$. Note that each element $\gamma$ of $\Gamma$ induces an evaluation map $\tau_{\gamma}: X(\Gamma)_{\mathbb{F}} \longrightarrow \mathbb{F}$ defined as $\tau_{\gamma}(\chi)=\chi(\gamma)$.

For simplicity, when $\mathbb{F}=\mathbb{C}$ we will usually write $X(\Gamma)$ instead of $X(\Gamma)_{\mathbb{C}}$.

Proposition $5([6,8])$. The set of characters $X(\Gamma)$ is an affine algebraic set defined over $\mathbb{Z}$, which embeds in $\mathbb{C}^{N}$ with coordinate functions $\left(\tau_{\gamma_{1}}, \ldots, \tau_{\gamma_{N}}\right)$ for some $\gamma_{1}, \ldots, \gamma_{N} \in \Gamma$.

The affine algebraic set $X(\Gamma)$ is called the character variety of $\Gamma$ : it can be interpreted as the algebraic quotient of the variety of representation of $\Gamma$ into $\mathrm{SL}_{2}(\mathbb{C})$ by the conjugacy action of $\mathrm{PSL}_{2}(\mathbb{C})=\mathrm{SL}_{2}(\mathbb{C}) / \mathcal{Z}\left(\mathrm{SL}_{2}(\mathbb{C})\right)$.

Note that the set $\left\{\gamma_{1}, \ldots, \gamma_{N}\right\}$ in the above proposition can be chosen to contain a generating set of $\Gamma$. For $\Gamma$ the fundamental group of a knot exterior, we will then assume that it always contains a representative of the meridian.

It is worth pointing out that the polynomial equations with integer coefficients of Proposition [5 only determine $X(\Gamma)$ as an algebraic set and not as a scheme.

A careful analysis of the arguments in [8] shows that Proposition 5 still holds if $\mathbb{C}$ is replaced by any algebraically closed field $\mathbb{F}$, provided that its characteristic is different from 2 . Let $\mathbb{F}_{p}$ denote the field with $p$ elements and $\overline{\mathbb{F}}_{p}$ its algebraic closure. We have:

Proposition 6 (8). Let $p>2$ be an odd prime number. The set of characters $X(\Gamma)_{\mathbb{F}_{p}}$ associated to representations of $\Gamma$ into $\mathrm{SL}_{2}\left(\overline{\mathbb{F}}_{p}\right)$ is an algebraic set which embeds in $\overline{\mathbb{F}}_{p}^{N}$ with coordinate functions $\left(\tau_{\gamma_{1}}, \ldots, \tau_{\gamma_{N}}\right)$ for the same $\gamma_{1}, \ldots, \gamma_{N} \in \Gamma$ seen in Proposition 5. Moreover, $X(\Gamma)_{\mathbb{F}_{p}}$ is defined by the reductions mod $p$ of the polynomials with coefficients in $\mathbb{Z}$ which define $X(\Gamma)$.

The key observation here is that, as in the case of complex numbers, the algebraic set $X(\Gamma)_{\overline{\mathbb{F}}_{p}}$ can be again interpreted as the algebraic quotient of the variety of representation of $\Gamma$ into $\mathrm{SL}_{2}\left(\overline{\mathbb{F}}_{p}\right)$ by the conjugacy action of $\mathrm{PSL}_{2}\left(\overline{\mathbb{F}}_{p}\right)=\mathrm{SL}_{2}\left(\overline{\mathbb{F}}_{p}\right) / \mathcal{Z}\left(\mathrm{SL}_{2}\left(\overline{\mathbb{F}}_{p}\right)\right)$

We will be mainly interested in the situation where $\Gamma$ is the fundamental group $\pi_{1}\left(\mathbf{S}^{3} \backslash K\right)$ of the exterior of a knot $K$. In this case we shall write $X(K)_{\mathbb{F}}$ or $X(K)$ instead of $X\left(\pi_{1}\left(\mathbf{S}^{3} \backslash K\right)\right)_{\mathbb{F}}$ or $X\left(\pi_{1}\left(\mathbf{S}^{3} \backslash K\right)\right)$ respectively.

Since the abelianisation of a knot group is infinite cyclic, for each element $A$ of $\mathrm{SL}_{2}(\mathbb{F})$ there is precisely one abelian representation of $\pi_{1}\left(\mathbf{S}^{3} \backslash K\right)$ defined by sending a meridian of $K$ to $A$. The set of abelian representations projects onto a rational, one dimensional component, of $X(K)_{\mathbb{F}}$.

If $K$ is a hyperbolic knot, $\pi_{1}\left(\mathbf{S}^{3} \backslash K\right)$ admits a hyperbolic holonomy representation into $\mathrm{PSL}_{2}(\mathbb{C})$. It is not hard to see that such representation lifts to a representation $\pi_{1}\left(\mathbf{S}^{3} \backslash K\right) \longrightarrow \mathrm{SL}_{2}(\mathbb{C})$. It was proved by Thurston that the character of this representation is a smooth point on a one-dimensional irreducible component of $X(K)$, known as the canonical or excellent component of $K$. More generally, Thurston proved that every irreducible component of $X(K)$ has dimension at least one $13,25$.

\section{Characters vanishing at the meridian}

Consider the map

$$
t=\tau_{\mu}: X(K) \rightarrow \mathbb{C}
$$


sending a character to the trace of the meridian. We are interested in its fiber at 0 :

$$
Y:=t^{-1}(0) \subset X(K) .
$$

Note that if we choose the trace of the meridian to be one of the coordinates of $X(K)$, the map is just a projection onto a coordinate and the fibre the intersection of $X(K)$ with a hyperplane. The condition $t\left(\chi_{\rho}\right)=0$ means that $\chi_{\rho}$ is the character of a representation $\rho$ that maps $\mu$ to a matrix $A$ such that $A^{2}=-$ Id. Consider the induced representation $\hat{\rho}$ obtained by composing $\rho$ with the natural quotient $\mathrm{SL}_{2}(\mathbb{C}) \longrightarrow \mathrm{PSL}_{2}(\mathbb{C}): \hat{\rho}$ restricts to a representation $\hat{\rho}^{\prime}$ of the double branched covering, $M_{2}$, of $K$.

We decompose the set $Y=t^{-1}(0)$ into three (possibly empty) disjoint subsets, according to the behaviour of $\hat{\rho}^{\prime}$

$$
Y=Y^{t r i v}(K) \sqcup Y^{a b}(K) \sqcup Y^{n a b}(K),
$$

where:

- $\chi \in Y^{\operatorname{triv}}(K)$ if there is a representation $\rho$ with character $\chi$ such that $\hat{\rho}^{\prime}$ is trivial;

- $\chi \in Y^{a b}(K)$ if there is a representation $\rho$ with character $\chi$ such that $\hat{\rho}^{\prime}$ is abelian, but no such abelian representation is trivial;

- $Y^{n a b}(K)$ if every representation $\rho$ with character $\chi$ is such that $\hat{\rho}^{\prime}$ is not abelian.

Note that $Y^{\text {triv }}(K)=X^{a b}(K) \cap Y$ always consists of a single point, the character of the abelian representation in $t^{-1}(0)$.

In characteristic zero, $Y^{a b}(K)$ is the set of binary dihedral representations and also the set of metabelian (and non-abelian) representations (see [14, 17]). As the cardinality of $H_{1}\left(M_{2}, \mathbb{Z}\right)$ is $\left|\Delta_{K}(-1)\right|$, an odd integer, the cardinality of $Y^{a b}(K)$ is

$$
\left|Y^{a b}(K)\right|=\frac{\left|\Delta_{K}(-1)\right|-1}{2} .
$$

Thus $Y^{a b}(K)=\emptyset$ if and only if $\Delta_{K}(-1)= \pm 1$.

Note that the subset $Y^{t r i v}(K) \cup Y^{a b}(K)$ is a subvariety of $Y(K)$ and thus of $X(K)$. Indeed, this set corresponds to the fibre $t^{-1}(0)$ of $X(\Gamma)$, where $\Gamma$ is the quotient of $\pi_{1}\left(\mathbf{S}^{3} \backslash K\right)$ defined in the introduction.

Before describing the reduction mod $p$ of $Y^{a b}(K)$ we need a lemma on finite order elements of $\mathrm{PSL}_{2}(\mathbb{K})$. Its proof is a straightforward consequence of the structure of $\mathrm{PSL}_{2}\left(\mathbb{F}_{p^{k}}\right)$ (see [7]).

Lemma 7. Let $\mathbb{F}$ be an algebraically closed field of characteristic $p \geq 3$ and $A \in \mathrm{PSL}_{2}(\mathbb{F})$ an element of finite order. Then either $\operatorname{order}(A)$ is coprime with $p$ or $\operatorname{order}(A)=p$. In addition:

- When $\operatorname{order}(A)$ is coprime with $p$, then $A$ is diagonalisable.

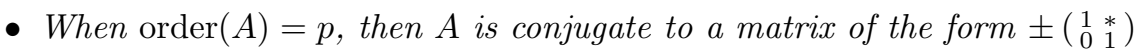

Corollary 8. For any prime $p$, write $\Delta_{K}(-1)= \pm p^{k} m$, with $m \geq 1$ an integer coprime with $p$. Then the reduction $\bmod p$ of $Y^{a b}(K)$ has cardinality $\frac{m-1}{2}$. 
The previous corollary is an example of collapse when reducing $\bmod p$. Notice that when $m=1$, i.e. when $\Delta_{K}(-1)= \pm p^{k}$, the reduction $\bmod p$ of $Y^{a b}(K)$ collapses to a single point, the abelian character in $Y^{\text {triv }}(K)$. Note that, in characteristic $p$, this is now also the character of a reducible dihedral representation.

Regarding $Y^{n a b}(K)$, we are interested in the following cases for $K$ :

1. $K$ a two bridge knot. Then $Y^{n a b}(K)=\emptyset$ because $M_{2}$ is a lens space.

2. $K$ is a Montesinos knot. Then, as $M_{2}$ is Seifert fibred, $Y^{n a b}(K)$ consists of representations whose projections into $\mathrm{PSL}_{2}(\mathbb{C})$ factor trough the base 2-orbifold of the Seifert fibration. In particular for pretzel knots $Y^{n a b}(K)$ is finite, because the variety of characters of triangle groups has finite cardinality.

3. For $\pi$-hyperbolic knots $Y^{n a b}(K)$ contains the lift of the holonomy of the orbifold with cone angle $\pi$. This is an isolated point of $Y(K)$ (Weil local rigidity), but there may be more characters in $Y^{n a b}(K)$.

\section{Two-bridge knots}

Let $K$ be a 2-bridge knot. Recall that $K$ is determined by its 2 -bridge notation, a rational number of the form $\beta / \alpha$, with $\alpha$ odd: one can recover a four-plat description of $K$ from a continued fraction expansion for $\beta / \alpha$, and the resulting knot does not depend on the chosen expansion. Moreover the 2-fold branched cover $M_{2}$ of $K$ is precisely the lens space $L(\alpha, \beta)$ (see [3. Ch 12]).

The fundamental group $\pi_{1}\left(\mathbf{S}^{3} \backslash K\right)$ of a 2-bridge knot $K$ admits a presentation of the form $\langle a, b \mid a w=w b\rangle$, with $a$ and $b$ meridians and $w$ a word in the generators, $a$ and $b$, and their inverses.

Since $a$ and $b$ are conjugate, the character variety $X(K)$ of $K$ is a plane curve with coordinates $t=\tau_{a}=\tau_{b}$ and $x=\tau_{a b^{-1}}$ :

$$
\left\{(t, x) \in \mathbb{C}^{2} \mid P(t, x)(x-2)=0\right\},
$$

where $x=2$ is the set of characters of abelian representations, and its complement consists of characters of irreducible representations.

Notice that $P$ is an even polynomial of the variable $t$ (i.e. a polynomial in $t^{2}$ ): there is a natural action of $H^{1}\left(S^{3}-K ; \mathbb{Z} / 2\right) \cong \mathbb{Z} / 2$ on the variety of characters, that maps a point with coordinates $(t, x)$ to $(-t, x)$. By taking $T=t^{2}$ as a new variable, this defines the variety of $\mathrm{PSL}_{2}(\mathbb{C})$-characters $[8,10$.

Because of their simple structure, character varieties of 2-bridge knots have been widely studied, see for instance [10, 19, 18, 15, 4], see also [12] for 2-bridge links.

Here, again, we are interested in $Y(K)$. We have:

$$
P(0, x)=\Phi_{\alpha}(x), \quad \text { where } \Phi_{\alpha}\left(\lambda+\frac{1}{\lambda}\right)=\frac{\lambda^{\alpha}-1}{\lambda-1} \lambda^{\frac{1-\alpha}{2}} .
$$

Namely, the factors of $\Phi_{\alpha}$ define the intersection with $\mathbb{R}$ of the corresponding cyclotomic extension.

Lemma 9. For $p \mid \alpha$, write $\alpha=p^{r} \alpha^{\prime}$, with $\alpha^{\prime} \in \mathbb{Z}$ coprime with $p$. 
1. $P(0, x)=0$ consists of $\frac{\alpha-1}{2}$ smooth points in $\mathbb{C}$.

2. The reduction mod $p$ of $P(0, x)=0$ consists of $\frac{\alpha^{\prime}-1}{2}$ points in $\overline{\mathbb{F}_{p}}$.

3. If $p^{r} \neq 3$, then the intersection between $P(t, x)=0$ and $x=2 \bmod p$ is not transverse.

Proof. The assertions on the cardinality are proved in the previous corollary. Smoothness is a consequence from the fact that the roots of $\Phi_{\alpha}$ are simple. It is also proved in [2, Lemmas 4.4 and 4.5].

In terms of the polynomial $\Phi_{\alpha}$ we have:

$$
\Phi_{\alpha}(x) \equiv(x-2)^{\frac{p^{r}-1}{2}}\left(\Phi_{\alpha}(x)\right)^{p^{r}} \bmod p
$$

For assertion 3) use that

$$
P(t, x)=\Phi_{\alpha}(x)+t^{2} Q\left(t^{2}, x\right)
$$

and the previous congruence of $\Phi_{\alpha}(x) \bmod p$.

Remark 1. One may ask whether this order-1 ramification phenomenon is a consequence of an order-0 ramification phenomenon that occurs at the level of the character variety $X(K)$, namely that different irreducible components of $\left\{(t, x) \in \mathbb{C}^{2} \mid P(t, x)=0\right\}$ get identified together.

More specifically, for $\alpha=p$ we may ask whether $P(t, x)$ can be a power mod $p$. As the characteristic $p$ is odd, if $P(t, x)$ were a power mod $p$, then it would be the power of an even polynomial in $t$. However in [10, Proposition 5.2] it is shown that the degree of $P(t, x)$ in $t^{2}$ is strictly less than the degree of $P(0, x)$, so this can never be the case.

Remark 2. Let $D$ be discriminant of $\Delta_{K}(t)$. Assume that $D \neq 0$. Then, in characteristic zero, all roots of $\Delta_{K}(t)$ are simple, and by 9 the intersection of the abelian component $x=2$ with $P(t, x)=0$ is everywhere transverse. In characteristic $p$, if $p \nmid D$, then the intersection is still transverse, in particular the points $(t, 2)$ are smooth points of $P(t, x)=0$. When $p \mid D$, new singularities or tangencies with $x=2$ may appear at those points. Notice also that $\Delta_{K}(-1) \mid D$.

In some instances, we may also count how many components $X(K)$ has:

Lemma 10. If $\alpha$ is prime, then $P(t, x)$ is irreducible over $\mathbb{Q}$. If in addition $\frac{\alpha-1}{2}$ is also prime, then it is irreducible over $\mathbb{C}$.

Proof. The curve of characters $X(K)$ has no ideal points with $t=0$, by 1, Lemma A.1.1], hence every irreducible component of $X(K)$ intersects the line $t=0$ in at least one point. Thus, to prove that $P$ is irreducible (over $\mathbb{Q}$ or over $\mathbb{C})$ we need to check that all points in

$$
Y^{a b}(K)=\{(0, x) \mid P(0, x)=0\}
$$

are contained in the same irreducible component (recall that here $Y^{n a b}(K)=\emptyset$ ). As $P(0, x)=\Phi_{\alpha}(x)$ is irreducible over $\mathbb{Q}$, because $\alpha$ is prime, $P=0$ has only one component over $\mathbb{Q}$, so $P$ is irreducible over $\mathbb{Q}$.

For the second assertion, we notice first that at least two points of $Y^{a b}(K)$ lie in the same component over $\mathbb{C}$. Those points correspond to the lifts of 
the spherical holonomy of the orbifold with cone angle $\pi$. More precisely, the universal covering of $S O(4)$ is $S U(2) \times S U(2)$, and the lift of the holonomy yields two different characters in $Y^{a b}(K)$, one for the projection to each factor $S U(2)$, see for instance [11. In addition, there is a curve of smooth points that joins both characters [1, 26, 24, 23, coming from holonomies of spherical and Euclidean cone structures, hence they lie in the same irreducible component.

Once we know that two points of $Y^{a b}(K)$ lie in the same component, we consider the action of the Galois group. If $P$ decomposes in $\mathbb{C}$, it decomposes with coefficients in a number field $\mathbb{K}$, that we may assume to contain the real cyclotomic field (containing the solutions of $\Phi_{\alpha}(z)$ ). The Galois group $\operatorname{Gal}(\mathbb{K} \mid \mathbb{Q})$ acts on $P=0$ by preserving or permuting irreducible components, in particular if $P=0$ contains more than one irreducible component, then $Y^{a b}(K)$ has a nontrivial partition induced by the components of $P=0$. As $\operatorname{Gal}(\mathbb{K} \mid \mathbb{Q})$ acts transitively on $Y^{a b}(K)$, all subsets of this partition have the same cardinality, which must be either 1 or $(\alpha-1) / 2$, because $(\alpha-1) / 2$ is prime. It cannot be 1 because we showed in the previous paragraph that the cardinality is at least 2 . Hence it is $(\alpha-1) / 2$ and there is a single component.

The reader should compare this result with [18] where a condition is provided for the character variety of a 2-bridge knot to have at least two irreducible components, besides the abelian one.

In the following we provide some examples of character varieties of 2-bridge knots, for different values of $\alpha$, notably $\alpha$ a prime number, a power of a prime number, and a product of different primes, in order to cover potentially different kinds of behaviour. In the first three instances, we also compute all ramified primes.

\section{EXAMPLE: $6_{1}=7 / 9$}

From the presentation

$$
\pi_{1}\left(\mathbf{S}^{3} \backslash 6_{1}\right)=\left\langle\alpha, \beta \mid \beta \alpha^{-1} \beta \alpha \beta^{-1} \alpha^{-1} \beta \alpha \beta^{-1}=\alpha^{-1} \beta \alpha \beta^{-1} \alpha^{-1} \beta \alpha \beta^{-1} \alpha\right\rangle,
$$

using coordinates

$$
t=\operatorname{tr}_{\alpha}=\operatorname{tr}_{\beta}, \quad x=\operatorname{tr}_{\alpha \beta^{-1}},
$$

and taking traces of the terms in the relation, we easily compute $P(t, x)$ :

$$
t^{4} x^{2}-3 t^{4} x-2 t^{2} x^{3}+2 t^{4}+2 t^{2} x^{2}+x^{4}+5 t^{2} x+x^{3}-4 t^{2}-3 x^{2}-2 x+1
$$

Even if 9 is not prime, $P(t, x)$ is irreducible over $\mathbb{C}$, as we will see in a while. We give here a reason why it is irreducible over $\mathbb{Q}$. We reproduce the argument in the proof of Lemma 10, setting $t=0$, we have

$$
P(0, x)=\Phi_{9}(x)=\Phi_{3}(x) \psi(x),
$$

where the solution of $\Phi_{3}(x)=x+1=0$ is -1 , twice the real part of the primitive roots of unity of order 3 , and the solutions of $\psi(x)=x^{3}-3 x+1=0$ are twice the real parts of primitive roots of unity of order 9, see (1). Thus, as there is no ideal point with $t=0$ [1, Lemma A.1.1], to prove irreducibly over $\mathbb{Q}$ we must show that there are two points in the same component, with coordinates $(0,-1)$ and $\left(0, x_{2}\right)$, with $\psi\left(x_{2}\right)=0$. Following the argument of Lemma 10 . 
those points are provided by the lift to $\mathrm{SU}(2) \times \mathrm{SU}(2)$ of the holonomy of the spherical orbifold. Up to conjugation, the lift of the holonomy of $\pi_{1}\left(M_{2}\right)$ is a cyclic group generated by

$$
\left(\left(\begin{array}{cc}
e^{i \theta_{1} / 2} & 0 \\
0 & e^{i \theta_{1} / 2}
\end{array}\right),\left(\begin{array}{cc}
e^{i \theta_{2} / 2} & 0 \\
0 & e^{i \theta_{2} / 2}
\end{array}\right)\right)
$$

By the description of the action of $\mathrm{SU}(2) \times \mathrm{SU}(2)$ on $\mathrm{SU}(2) \cong S^{3}$ [16], and as $M_{2} \cong L(9,7), \theta_{1}$ and $\theta_{2}$ must satisfy

$$
\theta_{2}-\theta_{1}=\frac{1}{9} 2 \pi, \quad \theta_{1}+\theta_{2}=\frac{7}{9} 2 \pi
$$

Hence

$$
\theta_{1}=\frac{1}{3} 2 \pi \quad \theta_{2}=\frac{2}{9} 2 \pi,
$$

and $x_{1}=2 \cos \theta_{1}=-1$ and $x_{2}=2 \cos \theta_{2}$ are the values we are looking for.

Observe also that the action of the Galois group permutes the points $\left(0, x_{2}\right)$ with $x_{2}^{3}-3 x_{2}+1=0$, while fixing the point $(0,-1)$, so there is a single component over $\mathbb{C}$.

As just observed, for $t=0$ we have:

$$
P(0, x)=\Phi_{3}(x) \psi(x)=(x+1)\left(x^{3}-3 x+1\right) .
$$

According to Theorem $1 Y(K)$ collapses to a single point mod 3, because

$$
P(0, x) \cong(x-2)^{4} \quad \bmod 3 .
$$

Note that the curve of irreducible characters meets the line of abelian ones in two points with multiplicity 2: these are computed as the solutions of the equation $0=P(t, 2)=-2 t^{2}+9$. The two components also have an intersection at infinity, also of multiplicity 2. The two (finite) intersections collapse to a single one in characteristic $p=3$ (and in this characteristic only), and the two components become tangent. The latter assertion is easily seen by rewriting $P(t, x)=t^{4}\left[(x-2)^{2}+(x-2)\right]+t^{2}\left[2(x-2)^{3}-10(x-2)^{2}+11(x-2)+42\right]+$ $(x+1)\left[(x-2)^{3}+6(x-2)^{2}+9(x-2)+3\right]$ so that one also observes that the component is not smooth at this point in characteristic $p=3$.

We want to show that $p=3$ is the only ramified prime for the character variety of the knot $66_{1}$. Clearly, for no prime the dimension of the variety can decrease or increase and it will always consists of at least two irreducible components. We will prove that the component defined by $P(t, x)=0$ is smooth over $\mathbb{C}$, and so is the corresponding component in any characteristic $p>3$.

The partial derivatives are

$$
\partial_{t} P(t, x)=2 t\left[t^{2}\left(2 x^{2}-6 x+4\right)-2 x^{3}+2 x^{2}+5 x-4\right]
$$

and

$$
\partial_{x} P(t, x)=t^{4}(2 x-3)+t^{2}\left(-6 x^{2}+4 x+5\right)+4 x^{3}+3 x^{2}-6 x-2 .
$$

Assume first that $t=0$. In this case we need to find the common solutions of $P(0, x)=0$ and $\partial_{x} P(0, x)=0$ : the discriminant of $P(0, x)$ is $3^{6}$ so these 
two polynomials have a common root only if $p=3$ and we already know what happens in that case.

We can thus assume that $t \neq 0$. Consider now the polynomial $\left(2 x^{2}-6 x+4\right)$ : its roots are 1 and 2 . A computation shows that $\partial_{t} P(t, 1)$ and $\partial_{t} P(t, 2)$ cannot be zero in characteristic 0 or odd.

We can thus multiply $\partial_{x} P(t, x)$ times $\left(2 x^{2}-6 x+4\right)^{2}$ in order to eliminate $t$, and we get $\partial_{x} P(t, x)\left(2 x^{2}-6 x+4\right)^{2}=3 x^{2}-4 x$.

Assume $x=0$. In this case $t^{2}=1$ and $P(1,0)=-1$, so this case cannot arise.

If $p=3$, then again we must have $x=0$ and the preceding reasoning applies.

We can thus assume that $p \neq 3$ and $x=4 / 3$ and $t^{2}=10 / 9$ and again $P(4 / 3,10 / 9)$ can never be 0 for any $p$.

We now will show that the variety consists of precisely two irreducible components in every characteristic $(\neq 2)$. Obviously the abelian component, being a line, can never split into more than one component. It is thus enough to prove that $P(t, x)$ is always irreducible. Assume by contradiction that curve corresponding to $P(t, x)=0$ is not irreducible. Its irreducible components must intersect in the projective closure in some singular points, since we are considering algebraically closed fields. Similarly, every irreducible component must meet the line at infinity. We now observe that our curve intersects the line at infinity in two points, one with (homogeneous) coordinates equal to $t=1$ and $x=0$ and a second one with coordinates $t=0$ and $x=1$. The first point has multiplicity four and is smooth with tangent the line at infinity in any characteristic $\neq 2$. The second one has multiplicity two and is a singular point, again with tangent the line at infinity. Since the first point is smooth, it must belong to a single irreducible component. If $p \neq 3$ this irreducible component must also contain the second point at infinity, which is the only singular point of the curve. Because of the multiplicities of intersection, this irreducible component must be a curve of degree at least five. It follows that if there were a second component it should be a line, passing through the second point and tangent to the line at infinity. This is however absurd since the component cannot be the line at infinity. If $p=3$, the component containing the smooth point at infinity might have degree 4 , since there are two singular points. We consider then its intersection with the line of equation $x=2$. Because of its degree, this component meets the line at the singular point $(t=0, x=2)$. If the multiplicity of intersection were 4 , then the component could not intersect the line elsewhere and thus it could not intersect the remaining irreducible component(s) since it could not pass through the second singular point which is also on this line. This shows that the component must pass through the second point at infinity even in this case and the conclusion follows as in the previous one.

$$
\text { ExAmPle: } 4_{1}=4 / 5
$$

We already discussed the character variety of the figure-eight knot in the introduction. It is well-known that it consists of just two irreducible components: the abelian one and Thurston's excellent one. This is also a consequence of Proposition 4 since both $\alpha=5$ and $(\alpha-1) / 2=2$ are prime numbers.

The same type of arguments and computations used in the previous example, show that in this case, too, there is a single odd prime for which the character 
variety ramifies, that is $p=5$. Recall that in this case we have

$$
P(t, x)=\left(x^{2}+\left(1-t^{2}\right) x+t^{2}-1\right)
$$

while the partial derivatives are

$$
\partial_{t} P(t, x)=2 t[x-1]
$$

and

$$
\partial_{x} P(t, x)=2 x-t^{2}+1 .
$$

We see that singular points can only occur for $t=0$ or $x=1$. The latter case, is impossible as $P(t, 1)=1$ for any $t$. In the former case we must have $2 x+1=0$ or equivalently $x=-1 / 2$ since we are assuming $p \neq 2$. This gives $P(t, x)=-5 / 4$, so we have singular points only for $p=5$. This is precisely the situation described by Theorem 1 .

To see that the reduction mod $p$ of the polynomial $P(t, x)$ is irreducible for all $p \geq 3$, one observes that the points at infinity of $P(t, x)$ are both smooth. As a consequence, $P(t, x)$ can only split if $p=5$ and at most into two components. Since the degrees of the two irreducible components must be 2 and 1 , the component of degree one is necessarily one of the tangents at the singular point $(t=0, x=1)$, however the two tangents do not meet the line at infinity at the same points as $P(t, x)$. The computational details are left to the reader.

$$
\text { EXAMPLE: } 7_{4}=11 / 15
$$

The character variety of this twist knot was computed and studied in [4] by $\mathrm{Chu}$ who was interested in understanding intersections between different, non abelian, irreducible components. Indeed, in this case the variety has two irreducible components, besides the abelian one, as shown by [18, 15].

Rewriting the equations in [4 using our notation, we have

$$
P(t, x)=\left(-1+2 x^{2}+x^{3}-x^{2} t^{2}\right)\left(1+4 x-4 x^{2}-x^{3}+x^{4}-2 x t^{2}+3 x^{2} t^{2}-x^{3} t^{2}\right) .
$$

Theorem 1 tells us that $p=3$ and $p=5$ are ramified primes for this variety. We wish to show that these are the only primes that ramify.

We start by studying each irreducible component separately. We begin with

$$
P_{1}(t, x)=-1+2 x^{2}+x^{3}-x^{2} t^{2}
$$

whose partial derivatives are

$$
\partial_{t} P_{1}(t, x)=-2 t x^{2}
$$

and

$$
\partial_{x} P_{1}(t, x)=-2 x t^{2}+4 x+3 x^{2} .
$$

We see that both derivatives are 0 if $x=0$ but $P_{1}(t, 0)=-1$, so we can exclude the value $x=0$. It follows that we must have $t=0$ and $4+3 x=0$. If $p=3$ there is no solution, so we can assume $p \neq 3$ and $x=-4 / 3$. We get $P_{1}(0,-4 / 3)=-5 / 27$ so, as expected, $p=5$ is a ramified prime. Writing $P_{1}(t, x)$ as $-t^{2}(x-2)^{2}-4 t^{2}(x-2)-4 t^{2}+(x-2)^{3}+8(x-2)^{2}+20(x-2)+50$, one sees that, in characteristic $p=5$, the tangent at the singular point $t=0$, 
$x=-4 / 3 \equiv 2$ has equation $t^{2}-2(x-2)^{2}=0$, where coefficients are thought $\bmod 5$.

To understand whether this component splits in some characteristic, we now analyse its points at infinity. There are two such points, both intersecting the line at infinity with multiplicity 2 . One of them, with homogeneous coordinates $t=0$ and $x=1$, is smooth with tangent the line at infinity, while the other, with homogeneous coordinates $t=1$ and $x=0$, is singular with tangent of equation $-x^{2}=0$. Consider now the irreducible component containing the smooth point at infinity. Since the curve only meets the line of equation $x=0$ at infinity, such irreducible component passes through both points at infinity and had degree at least 3 . If it had degree 3 , the second irreducible component would have degree one and would have to coincide with the tangent at the singular point, that is the line of equation $x=0$. This is clearly impossible, so this curve is irreducible in all characteristics.

We pass now to the second component

$$
P_{2}(t, x)=1+4 x-4 x^{2}-x^{3}+x^{4}-2 x t^{2}+3 x^{2} t^{2}-x^{3} t^{2}
$$

whose partial derivatives are

$$
\partial_{t} P_{2}(t, x)=2 t\left(-2 x+3 x^{2}-x^{3}\right)
$$

and

$$
\partial_{x} P_{2}(t, x)=4-8 x-3 x^{2}+4 x^{3}+t^{2}\left(-2+6 x-3 x^{2}\right) .
$$

From the first derivative, we see that either $x \in\{0,1,2\}$ or $t=0$. For each value of $x$ in $\{0,1,2\}$ we get $P_{2}(t, x)=1$, so we must have $t=0$. Computing the resultant of the polynomials $1+4 x-4 x^{2}-x^{3}+x^{4}$ and $4-8 x-3 x^{2}+4 x^{3}$ one obtains $3^{2} 5^{3}$ : as expected the curve has singular points in characteristics $p=3$ and $p=5$.

As for the previous component, we show that this component as well is always irreducible. As before we consider the points at infinity of the curve: again we have two points. The first one has homogeneous coordinates $t=0$ and $x=1$, it is smooth with tangent the line at infinity, and the multiplicity of intersection between the curve and the line at infinity at this point is 2 . The second point has homogeneous coordinates $t=1$ and $x=0$, it is a singular point with tangent of equation $x^{3}=0$ and multiplicity of intersection 3 . The very same argument seen in the previous case, shows that the irreducible component containing the smooth point at infinity must pass through the second one as well and have degree at least 3 . If the curve splits in two or more irreducible components, the component of degree at most two must be pass through the singular point at infinity with tangent of equation $x=0$. As a consequence such component can only be the line $x=0$, with multiplicity one or two. This is however impossible.

We turn now our attention to the points of intersection between the two components. Since $x$ cannot be equal to 0 , we can use the $P_{1}(t, x)$ to eliminate $t^{2}$ from $P_{2}(t, x)$. We obtain the polynomial $x^{2}-2 x+2$ that always has two roots in any characteristic different from 2 (cfr. 4]).

EXAmPLE: $89=7 / 25$ 
From the presentation

$$
\pi_{1}\left(\mathbf{S}^{3} \backslash 8_{9}\right)=\left\langle\alpha, \beta \mid \beta \alpha^{-1} \beta \alpha \beta^{-1} \alpha^{-1} \beta \alpha \beta^{-1}=\alpha^{-1} \beta \alpha \beta^{-1} \alpha^{-1} \beta \alpha \beta^{-1} \alpha\right\rangle
$$

by taking traces and using the coordinates $t=\operatorname{tr}_{\alpha}=\operatorname{tr}_{\beta}, x=\operatorname{tr}_{\alpha \beta^{-1}}$ with the help of symbolic software we get

$$
\begin{aligned}
& P(t, x)=t^{18} x^{3}-6 t^{18} x^{2}-9 t^{16} x^{4}+12 t^{18} x+47 t^{16} x^{3}+36 t^{14} x^{5}-8 t^{18}-66 t^{16} x^{2}- \\
& 160 t^{14} x^{4}-84 t^{12} x^{6}-12 t^{16} x+115 t^{14} x^{3}+308 t^{12} x^{5}+126 t^{10} x^{7}+56 t^{16}+273 t^{14} x^{2}+ \\
& 35 t^{12} x^{4}-364 t^{10} x^{6}-126 t^{8} x^{8}-232 t^{14} x-928 t^{12} x^{3}-441 t^{10} x^{5}+266 t^{8} x^{7}+ \\
& 84 t^{6} x^{9}-140 t^{14}+187 t^{12} x^{2}+1515 t^{10} x^{4}+763 t^{8} x^{6}-112 t^{6} x^{8}-36 t^{4} x^{10}+ \\
& 756 t^{12} x+630 t^{10} x^{3}-1362 t^{8} x^{5}-679 t^{6} x^{7}+20 t^{4} x^{9}+9 t^{2} x^{11}+124 t^{12}-1648 t^{10} x^{2}- \\
& 1755 t^{8} x^{4}+647 t^{6} x^{6}+345 t^{4} x^{8}+2 t^{2} x^{10}-x^{12}-725 t^{10} x+1796 t^{8} x^{3}+1964 t^{6} x^{5}- \\
& 108 t^{4} x^{7}-95 t^{2} x^{9}-x^{11}+42 t^{10}+1744 t^{8} x^{2}-924 t^{6} x^{4}-1163 t^{4} x^{6}-27 t^{2} x^{8}+ \\
& 11 x^{10}-93 t^{8} x-2206 t^{6} x^{3}+76 t^{4} x^{5}+358 t^{2} x^{7}+10 x^{9}-118 t^{8}-29 t^{6} x^{2}+ \\
& 1544 t^{4} x^{4}+120 t^{2} x^{6}-45 x^{8}+422 t^{6} x+225 t^{4} x^{3}-565 t^{2} x^{5}-36 x^{7}+25 t^{6}- \\
& 560 t^{4} x^{2}-201 t^{2} x^{4}+84 x^{6}-85 t^{4} x+326 t^{2} x^{3}+56 x^{5}+25 t^{4}+95 t^{2} x^{2}-70 x^{4}- \\
& 46 x t^{2}-35 x^{3}-6 t^{2}+21 x^{2}+6 x-1
\end{aligned}
$$

It intersects $t=0$ at

$$
\left(x^{2}+x-1\right)\left(x^{10}-10 x^{8}+35 x^{6}+x^{5}-50 x^{4}-5 x^{3}+25 x^{2}+5 x-1\right)
$$

Which corresponds to roots of order 5 and 25, respectively. This polynomial is congruent to

$$
(x-2)^{12} \bmod 5
$$

Thus the whole $Y(K)$ collapses to a single point when reducing mod 5 .

Unexpectedly, $P(t, x)$ factors non trivially $\bmod 7$ (but not $\bmod 5$ ):

$$
\begin{aligned}
& P(t, x)=\left(t^{12} x^{2}\right.+3 t^{12} x+t^{10} x^{3}+4 t^{12}+6 t^{10} x^{2}+t^{8} x^{4}+6 t^{10} x+2 t^{8} x^{3}+t^{6} x^{5}+5 t^{10} \\
&+6 t^{8} x^{2}+5 t^{6} x^{4}+t^{4} x^{6}+6 t^{8} x+4 t^{6} x^{3}+t^{4} x^{5}+t^{2} x^{7}+2 t^{8}+t^{6} x^{2}+4 t^{2} x^{6} \\
&+x^{8}+4 t^{6} x+2 t^{4} x^{3}+t^{2} x^{5}+5 t^{6}+4 t^{4} x^{2}+t^{4} x+4 t^{4}+4 t^{2} x^{2}+4 x^{4}+5 x t^{2} \\
&\left.+4 x^{3}+5 t^{2}+5 x^{2}+2 x+6\right) \\
& \times\left(t^{6} x+5 t^{6}+4 t^{4} x^{2}+3 t^{4} x+3 t^{2} x^{3}+6 t^{4}+6 x^{4}+4 x t^{2}+6 x^{3}\right. \\
&\left.+4 t^{2}+4 x^{2}+3 x+1\right) \quad \bmod 7
\end{aligned}
$$

Using symbolic software again, we may check that in characteristic zero $P(t, x)=0$ has no singular points. In fact, besides characteristic $p=5$ and $p=7$, in characteristic $p=23$ one finds that $(t, x)=(2, \pm 6)$ is a singular point of the variety. Notice that the discriminant of the Alexander polynomial is $13225=5^{2} 23^{2}$, which, by Remark 2 , implies that there are singular points with $x=2$ in characteristic $p$ at most for $p=5$ and $p=23$.

EXAMPLE: $11 / 23$

In this case the order of the homology of the double branched cover is a prime number and moreover we have that $11=(23-1) / 2$ is also prime. We mainly 
study this twist knot because its character variety appears as a subvariety of the Whitehead double we study in Section 8 , Indeed, there is a degree-one map from the Whitehead double to this knot obtained by sending the trefoil companion onto the trivial knot. Of course the map induces a surjection of fundamental groups and so an injection of character varieties.

We give here a presentation obtained from a presentation of the Whitehead link $5_{1}^{2}$ by surgery on one of its two components. This will become handy when studying the Whitehead double.

We have for the Whitehead link

$$
\pi_{1}\left(\mathbf{S}^{3} \backslash 5_{1}^{2}\right)=\left\langle\alpha, b \mid \alpha b^{-1} \alpha^{-1} b \alpha^{-1} b^{-1} \alpha=b^{-1} \alpha b^{-1} \alpha^{-1} b \alpha^{-1} b^{-1} \alpha b\right\rangle,
$$

and for the twist knot

$\pi_{1}\left(\mathbf{S}^{3} \backslash 11 / 23\right)=\left\langle\alpha, \beta \mid u=\beta^{-1} \alpha, b=u^{6}, u=\alpha b^{-1} \alpha^{-1} b \alpha^{-1} b^{-1} \alpha b, u=b^{-1} u b\right\rangle$,

where the group of the twist knot is obtained by killing by Dehn surgery the slope $u^{6} b^{-1}$, where $u=\alpha b^{-1} \alpha^{-1} b \alpha^{-1} b^{-1} \alpha b$, so that the relation of the Whitehead link group becomes $u=b^{-1} u b$ which is redundant in the quotient group; finally one can recover a standard 2-bridge presentation for the twist knot by observing that $\beta=\alpha u^{-1}$ is conjugate to $\alpha$ hence a meridian that can be chosen as a generator of the group.

Hence we take coordinates

$$
t=\operatorname{tr}_{\alpha}=\operatorname{tr}_{\beta}, \quad x=\operatorname{tr}_{\alpha^{-1} \beta},
$$

and by taking traces on the equality

$$
\alpha^{-1}\left(\beta \alpha^{-1}\right)^{5} \beta \alpha\left(\beta^{-1} \alpha\right)^{5} \beta=\left(\beta \alpha^{-1}\right)^{5}(\beta \alpha)\left(\beta^{-1} \alpha\right)^{5}
$$

we get

$$
(x-2) P(t, x)=0
$$

where

$$
\begin{array}{r}
P(t, x)=t^{2} x^{10}-t^{2} x^{9}-x^{11}-8 t^{2} x^{8}-x^{10}+7 t^{2} x^{7}+10 x^{9}+22 t^{2} x^{6}+9 x^{8} \\
-16 t^{2} x^{5}-36 x^{7}-24 t^{2} x^{4}-28 x^{6}+13 t^{2} x^{3}+56 x^{5} \\
+9 t^{2} x^{2}+35 x^{4}-3 t^{2} x-35 x^{3}-15 x^{2}+6 x+1 .
\end{array}
$$

with

$$
P(0, x) \equiv-(x-2)^{11} \bmod 23
$$

An analysis similar to those seen for the first three examples shows that $p=23$ is the only prime that ramifies for this knot.

\section{A pretzel example}

For a Montesinos knot $K$ that is not a 2-bridge one, $Y^{n a b}(K) \neq \emptyset$, as it contains irreducible representations of the double branched cover $M_{2}$. Since $M_{2}$ is Seifert fibered, those representations from $\pi_{1}\left(M_{2}\right)$ to $\mathrm{PSL}_{2}(\mathbb{C})$ map the fibre to the identity (the centre of $\mathrm{PSL}_{2}(\mathbb{C})$ is trivial), hence they are irreducible representations of the 2-orbifold, the space of fibres. For a Montesinos knot with $k$ tangles, this will yield components in $Y^{n a b}(K)$ of dimension up to $k-3$, see for instance 20. For a pretzel knot, we have $k=3$. 
EXAMPLE: $8_{5}=P(3,3,2)$

Here $\Delta_{K}(-1)=21$, hence $\left|Y^{a b}(K)\right|=10$. If we restrict these characters to $M_{2}$, they are characters of ten cyclic representations $H_{1}\left(M_{2}, \mathbb{Z}\right) \rightarrow \mathrm{PSL}_{2}(\mathbb{C})$, one of them has order 3 , three have order 7 , and six have order 21 (the cardinality is one half of Euler's $\phi$ ).

Since $K$ is the pretzel $(2,3,3)$, elements in $Y^{n a b}(K)$ are characters of representations in $\mathrm{SU}(2)$.

We will consider reductions $\bmod p$ for $p=3,7$.

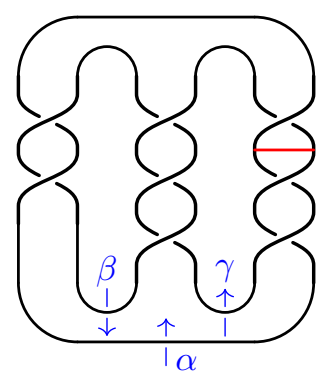

Figure 1: The knot $8_{5}$. The generators $\alpha, \beta$ and $\gamma$ of its fundamental group and a tunnel.

The group of $8_{5}$ is generated by three meridians, $\alpha, \beta$ and $\gamma$ in Figure 1 . Since it has tunnel number one, the group can also be generated by two elements. Those are $\gamma$ and $a=\gamma^{-1} \alpha^{-1}(\beta \gamma)^{2}$, see Appendix A. Hence we choose coordinates:

$$
\begin{aligned}
t & =\operatorname{tr}_{\gamma} \\
x & =\operatorname{tr}_{a} \\
y & =\operatorname{tr}_{\gamma a}
\end{aligned}
$$

With the help of symbolic software, there are 5 components:

1. Abelian component

$$
\begin{aligned}
& x=t^{2}-2 \\
& y=t^{3}-3 t,
\end{aligned}
$$

because $x$ and $y$ correspond to traces of elements whose abelianisations are 2 and 3 respectively.

2. A "trefoil" component:

$$
y=x-1=0
$$

namely, a component that looks like the variety of irreducible characters of the trefoil knot and which is induced by an epimorphism onto the trefoil group. 
3. The canonical component.

$$
\begin{aligned}
t x^{3}-t^{2} y-t y^{2}-x^{2} y+2 t x+2 y x-t-y & =0, \\
t^{2} y x+y^{2} t x-2 t x^{2}-t y^{2}-x^{2} y-y^{3}-t x+y x+t+2 y & =0, \\
t y^{3} x+t x^{2} y-y^{2} x^{2}-y^{4}-2 t x y-2 x^{3}-x y^{2}-t y+2 x^{2}+3 y^{2}+4 x-2 & =0 .
\end{aligned}
$$

4. An exotic component:

$$
x+1=t y+y^{2}-2=0
$$

5. A second exotic component:

$$
\begin{aligned}
& t^{2} x^{2} y-t x^{3}-2 y^{2} t x+x^{2} y+y^{3}+2 t x-t-3 y=0, \\
& t^{3} y x+t^{2} x y^{2}+t y^{3} x-t^{3} y-t^{2} x^{2}-2 t^{2} y^{2}-t x^{2} y-t y^{3}-y^{2} x^{2} \\
& -y^{4}+t^{2}+4 t y+4 y^{2}=0, \\
& t x^{5} y+t x^{4} y-x^{6}-x^{4} y^{2}+2 t^{2} x y^{2}-4 t y x^{3}+2 t y^{3} x-x^{5}-x^{3} y^{2} \\
& -t^{3} y-t^{2} y^{2}-7 t x^{2} y-2 t y^{3}+6 x^{4}+y^{2} x^{2}-2 y^{4}+t^{2} x+6 x^{3} \\
& +3 x y^{2}+t^{2}+8 t y-8 x^{2}+7 y^{2}-8 x-1=0
\end{aligned}
$$

Next we describe the intersection of each component with $t=0$ :

1. Abelian: $x=-2, y=0$. This is the point in $Y^{\text {triv }}(K)$

2. Trefoil: $y=0, x=1$. This point lies in $Y^{a b}(K)$ (it corresponds to the element of order 3 ).

3. Canonical: it is the union of two sets:

- $y=x^{3}-x^{2}-2 x+1=0$ that lies in $Y^{a b}(K)(3$ points of order 7$)$, and

- $x-1=y^{2}-2=0$, two points in $Y^{n a b}(K)$.

4. Exotic: $x+1=y^{2}-2=0$, two points in $Y^{n a b}(K)$.

5. Second Exotic: $y=-x^{6}-x^{5}+6 x^{4}+6 x^{3}-8 x^{2}-8 x-1=0$. Six points in $Y^{a b}(K)$ (of order 21).

Notice that the 4 points in $Y^{n a b}(K)$ correspond to two non conjugate representations in $\mathrm{PSL}_{2}(\mathbb{C})$ ( $x$ is already a variable of the characters in $\mathrm{PSL}_{2}(\mathbb{C})$ but $y$ is not).

This knot is $\pi$-spherical, so the lift of its holonomy in $\mathrm{SU}(2) \times \mathrm{SU}(2)$ projects to the tetrahedral group in one $\mathrm{SU}(2)$ factor (for the basis 2-orbifold), and to the binary dihedral group of order 28 in the other SU(2) factor. This dihedral group in one of the factors corresponds to an action of $\mathbf{S}^{1}$ that is preserved up to orientation, yielding the orbifold Seifert fibration.

Regarding the ramifications, we obviously have the collapses of $Y^{a b}(K) \bmod$ 3 and $\bmod 7$ :

- When reducing mod 3 , the point of order 3 becomes trivial (in $Y^{\text {triv }}(K)$ ). The 6 points of order 21 are identified to some of the 3 points of order 7 .

- When reducing $\bmod 7$, the points of order 7 become also trivial (in $\left.Y^{\text {triv }}(K)\right)$. The 6 points of order 21 become the point of order 3 . 


\section{A $\pi$-hyperbolic knot}

The knot $8_{18}$ is $\pi$-hyperbolic, that is its double branched cover is hyperbolic. Here the canonical component splits into two irreducible components in characteristic 3 .

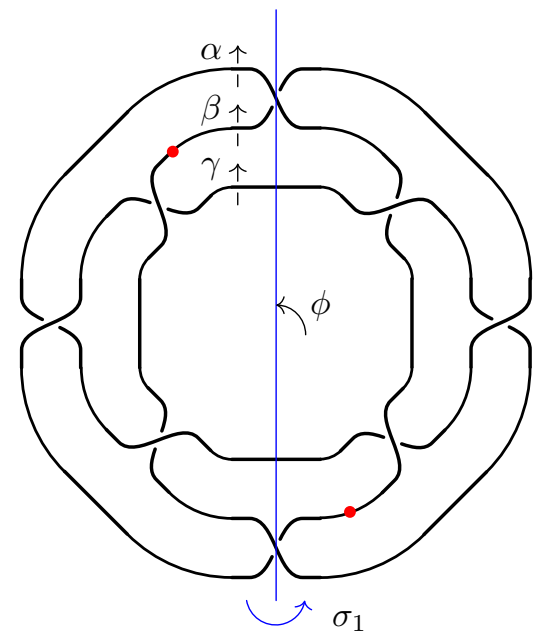

Figure 2: The knot $8_{18}$. The period $\phi$, the axis of the strong inversion $\sigma_{1}$ and the fixed points of the orientation reversing involution $\sigma_{2}$ marked as thick red dots

The knot $8_{18}$ has a period $\phi$, corresponding to the rotation of order four in Figure 2. The symmetry group of the knot is generated by the rotation together with two involutions $\sigma_{1}$ and $\sigma_{2}$. The orientation preserving involution $\sigma_{1}$ is a rotation of angle $\pi$ about an axis represented as a blue line in Figure 2 it is a strong inversion of the knot. The orientation-reversing involution $\sigma_{2}$ is reflection with two fixed points belonging to the knot (after a suitable conformal change of coordinates of $\mathbf{S}^{3}=\mathbb{R}^{3}$ sending one of the two fixed points to $\infty$ and the other to the origin, $\sigma_{2}$ can be seen as the linear map with matrix minus the identity).

Here $\left|\Delta_{K}(-1)\right|=45$, and $H_{1}\left(M_{2}\right)=\mathbb{Z} / 5 \oplus \mathbb{Z} / 3 \oplus \mathbb{Z} / 3$.

We sketch the computation of $X\left(8_{18}\right)$ in Appendix B, here we just describe it. We use the following coordinates for $X\left(8_{18}\right)$ :

$$
\begin{aligned}
& t=t r_{\alpha}=t r_{\beta}=t r_{\gamma} \\
& x=t r_{\alpha \beta^{-1}} \\
& y=t r_{\beta^{-1} \gamma} \\
& z=t r_{\alpha \gamma} \\
& w=t r_{\alpha \beta^{-1} \gamma}
\end{aligned}
$$

We next list the 11 components of $X\left(8_{18}\right)$.

\section{Abelian component}

$$
\left\langle-t+w, x-2, y-2,-t^{2}+z+2\right\rangle
$$


The symmetries act trivially here. When $t=0$ we get the character that is trivial on $M_{2}$, the double branched covering.

\section{The canonical component}

$$
\left\langle t w+y^{2}+\left(-t^{2}-1\right) y+1,-t^{2}+y+z, 5 t^{2}-4 t w+w^{2}-6,-t^{2}+x+y-1\right\rangle
$$

It can also be written as

$$
\begin{gathered}
t w+y^{2}+\left(-t^{2}-1\right) y+1=0 \\
z=t^{2}-y \\
x=z+1 \\
5 t^{2}-4 t w+w^{2}=6
\end{gathered}
$$

It is a double branched covering of the curve described in 12 obtained by considering only the variables $t$ and $y$ (we can get rid of $x$ and $z$, the projection is the elimination of $w$ ).

This component is fixed point-wise by the period $\phi$ and by $\sigma_{1}$. The involution $\sigma_{2}$ preserves the component but permutes the holonomy of the complete structure with its complex conjugate.

The intersection with $t=0$ yields precisely the 4 lifts of the hyperbolic holonomy of the orbifold $(t=0, y=(1 \pm \sqrt{-3}) / 2, w= \pm \sqrt{6})$. The 4 points correspond to complex conjugation $(y=(1 \pm \sqrt{-3}) / 2)$ and a choice of sign $(w= \pm \sqrt{6})$ as the abelianisation of the orbifold group is $\mathbb{Z} / 2$ (it only concerns the variable $w$ because $t=0$ ). When reducing $\bmod 3$, these 4 points become a single point.

It should be noticed that the last equation

$$
5 t^{2}-4 t w+w^{2}=6
$$

factors mod 3 as the product of two lineal equations, hence this component splits into 2 components when reducing mod 3 .

Four "trefoil-like" components There are four components that look like the the variety of characters of the trefoil:

$$
\begin{array}{r}
\left\langle-1+z,-t+w, x-2,-t^{2}+y+1\right\rangle \\
\left\langle-1+z,-t+w, y-2,-t^{2}+x+1\right\rangle \\
\left\langle-1+z,-t^{3}+2 t+w,-t^{2}+x+1,-t^{2}+y+1\right\rangle \\
\left\langle-t^{3}+2 t+w,-t^{2}+x+1,-t^{2}+y+1,-t^{2}+z+2\right\rangle
\end{array}
$$

They come from a surjection from $\pi_{1}\left(\mathbf{S}^{3} \backslash 8_{18}\right)$ to the group of the trefoil. Namely, if we add the relation $\alpha=\beta$ then we get the group of the trefoil, and this corresponds to the component (4).

For computational purposes, it is useful to give a parameterisation of these components:

$$
\begin{array}{llll}
x=2, & y=t^{2}-1, & z=1, & w=t,
\end{array}
$$


The period (the symmetry of order 4) permutes (4) with (6), and (5) with (77). The square of the period fixes point-wise these components.

The involution $\sigma_{1}$ preserves (4) and (6) and permutes (5) with (17).

The involution $\sigma_{2}$ permutes (4) and (5) and preserves the components (6) and (7). Thus the group of symmetries acts transitively on these components.

The intersection of the 4 components is the point $x=y=2, z=1, w=$ $t= \pm \sqrt{3}$, which also lies in the abelian component (the discriminant of the Alexander polynomial vanishes).

When $t=0$, we get the conjugacy classes of 4 representations of order 3 of $M_{2}$, as $H_{1}\left(M_{2}\right) \cong \mathbb{Z} / 3 \oplus \mathbb{Z} / 3 \oplus \mathbb{Z} / 5$.

The figure eight knot component This component looks like the character variety of the figure eight knot and comes from a surjection of fundamental groups. The figure eight knot is in fact the quotient of the $8_{18}$ by $\phi^{2}$, the square of the period. The ideal is:

$$
\left\langle-t+w, y^{2}+\left(-t^{2}+1\right) y+t^{2}-1,-t^{2}+y+z,-t^{2}+x+y+1\right\rangle
$$

It can also be presented as

$$
\begin{gathered}
w=t \\
(y-1) t^{2}=y^{2}+y-1 \\
z=t^{2}-y \\
x y=x+y
\end{gathered}
$$

It is point-wise invariant by the period and by the involution $\sigma_{1}$ and preserved by $\sigma_{2}$, but $\sigma_{2}$ permutes $x$ and $y$. In particular $\sigma_{2}$ swaps the holonomy of the figure eight knot with its complex conjugate.

When $t=0, y^{2}+y-1=0$ corresponds to the two conjugacy classes of nontrivial representations of $\mathbb{Z} / 5$.

Four more components When $t=0$, there are still 16 conjugacy classes of representations of $M_{2}$ of order 15 . They belong to 4 components. The first one is:

$$
\begin{aligned}
\left\langle x^{2}+\left(-t^{2}+1\right) x+\right. & t^{2}-1, \\
& \left(t^{2}-1\right) x+y-z-t^{4}+3 t^{2}-1 \\
\left(-t^{2}+1\right) x z+ & \left(t^{2}+1\right) x+z^{2}+\left(t^{4}-4 t^{2}+1\right) z-t^{4}+3 t^{2}-1 \\
& \left.-t^{3}+x t-z t+3 t+w\right\rangle
\end{aligned}
$$

The second one is:

$$
\begin{aligned}
& \left\langle y^{2}+\left(-t^{2}+1\right) y+t^{2}-1\right. \\
& w-x t+\left(-t^{3}+2 t\right) y+t^{5}-4 t^{3}+4 t \\
& x^{2}+\left(t^{2}-1\right) x y+\left(-t^{4}+2 t^{2}-1\right) x+\left(-t^{4}+2 t^{2}+1\right) y+t^{6}-4 t^{4}+4 t^{2}-1, \\
& \left.x y+\left(-t^{2}+1\right) x+\left(-t^{4}+3 t^{2}-1\right) y+z+t^{6}-5 t^{4}+6 t^{2}-1\right\rangle
\end{aligned}
$$


The third one

$$
\begin{aligned}
& \left\langle z^{2}+\left(-t^{2}-1\right) z+2 t^{2}-1\right. \\
& w+x z t-2 x t+\left(-t^{5}+4 t^{3}-4 t\right) z+2 t^{5}-7 t^{3}+5 t \\
& x^{2}+\left(-t^{2}+1\right) x z+\left(t^{2}-1\right) x+\left(t^{4}-2 t^{2}-1\right) z-2 t^{4}+5 t^{2}-1 \\
& \left.x z-x+y+\left(-t^{4}+3 t^{2}-1\right) z+2 t^{4}-6 t^{2}+1\right\rangle
\end{aligned}
$$

The last component is:

$$
\begin{gathered}
\left\langle t^{2}-y-z,\right. \\
-z x^{2}+x y^{2}+x y z+x z^{2}+x^{2}-4 x y-x z-2 y^{2}-y z-z^{2}+5 y+2 z-1, \\
x^{2} y z-x^{2} y-z x^{2}-4 x y z-x z^{2}+4 x y+6 x z+3 y z+z^{2}-x-2 y-5 z, \\
-x^{2} z^{2}+x z^{3}+2 z x^{2}+y z^{2}-z^{3}-x^{2}+x y-4 x z-2 y z+x-2 y+4 z+1, \\
z^{2} y x-4 x y z-2 x z^{2}+y^{2} z-y z^{2}+3 x y+6 x z-2 y^{2}+2 y z+2 z^{2}-2 x+2 y-5 z-1, \\
-x^{2} z^{2}+z^{2} y^{2}+2 z^{3} y+z^{4}+2 z x^{2}-2 x y z+x z^{2}-y^{2} z-7 y z^{2}-5 z^{3}-x^{2}+3 x y+x z \\
\quad-2 y^{2}+5 y z+6 z^{2}-2 x+4 y-z-1, \\
2 x^{2} z^{2}+z y^{3}-3 z^{3} y-2 z^{4}-4 z x^{2}+3 x y z-3 x z^{2}-2 y^{3}-8 y^{2} z+6 y z^{2}+9 z^{3}+2 x^{2}-5 x y \\
+16 y^{2}+10 y z-5 z^{2}+5 x-22 y-10 z+4, \\
\left.x z^{2} t-t y^{2} z-2 z^{2} y t-z^{3} t-2 x z t+2 t y^{2}+10 z y t+5 z^{2} t-12 t y-10 z t+7 t+w\right\rangle
\end{gathered}
$$

\begin{tabular}{|c|c|c|c|c|c|c|}
\hline & $\sigma_{1}$ & & $\sigma_{2}$ & \multicolumn{3}{|c|}{$\phi$} \\
\hline (99) & $\mapsto \quad 9$ & (9) & $\mapsto \quad$ (10) & (9) & $\mapsto$ & (12) \\
\hline 10 & $\mapsto \quad$ & (10) & (9) & 10 & $\mapsto$ & $(11)$ \\
\hline 11 & $\mapsto \quad$ (10 & (11) & $\mapsto \quad$ (12) & (11) & $\mapsto$ & (10) \\
\hline 11 & $\mapsto \quad(12$ & $(12)$ & $\mapsto \quad(11)$ & $(12$ & $\mapsto$ & 9]) \\
\hline
\end{tabular}

Each component intersects $t=0$ in 4 points, corresponding to representations of $M_{2}$ or order 15 . The action of the symmetry group on the components is:

So the four components are equivalent.

Characters of $Y\left(8_{18}\right)=Y^{\text {triv }}\left(8_{18}\right) \cup Y^{a b}\left(8_{18}\right) \cup Y^{n a b}\left(8_{18}\right)$

- $Y^{\text {triv }}\left(8_{18}\right)$ has a single point, with coordinates $t=w=0, x=y=2$, and $z=-2$

- $Y^{a b}\left(8_{18}\right)$ has $\frac{|\Delta(-1)|-1}{2}=22$ points. When looking at the order of the induced representation of $\pi_{1}\left(M_{2}\right)$ in $\mathrm{PSL}_{2}(\mathbb{C})$, they are distributed as follows: the 2 points of order 5 lie in the figure eight knot component, the 4 points of order 3 lie in trefoil components, one per component, and the 16 points of order 15 on the exotic components (4 per component). In characteristic $3, Y^{a b}\left(8_{18}\right)$ has $(5-1) / 2=2$ points, in characteristic 5 , $(9-1) / 2=4$ points.

- $Y^{n a b}\left(8_{18}\right)$ has 4 points, all of them in the canonical component: two lifts of the orbifold holonomy and their complex conjugate. 
When reducing mod 3: The 4 points of $Y^{a b}\left(8_{18}\right)$ in the trefoil components collapse to $Y^{\text {triv }}\left(8_{18}\right)$. The 2 points in the figure eight knot component remain as different points. On each exotic component, the 4 points become (the same) 2 points, that are also the 2 points for the figure eight component. The four points in $Y^{n a b}\left(8_{18}\right)$ become a single one, but different from the previous ones.

When reducing mod 5: The 4 points of $Y^{a b}\left(8_{18}\right)$ in the trefoil components remain as 4 different points. The four points of $Y^{a b}\left(8_{18}\right)$ in each exotic component become a single one, in fact the same as a point in one of the trefoil components, and points in different exotic components go to different trefoil components. The 2 points in the figure eight component collapse to $Y^{\text {triv }}\left(8_{18}\right)$. The 4 points in $Y^{n a b}\left(8_{18}\right)$ remain as different points.

\section{Torus Knots}

For $m \leq n \in \mathbb{N}$ coprime, the $(m, n)$-torus knot is denoted by $T(m, n)$. Since

$$
\pi_{1}\left(\mathbf{S}^{3} \backslash T(m, n)\right) \cong\left\langle a, b \mid a^{m}=b^{n}\right\rangle,
$$

irreducible representations map the central element $a^{m}=b^{n}$ to the centre $\{ \pm \mathrm{Id}\}$ (and only to - Id for $m=2$ ). Thus, besides the abelian one, the components of the variety of characters are determined by the conjugacy classes of elements in $\mathrm{SL}_{2}$ to which $a$ and $b$ are mapped. Therefore, when $p$ divides either $m$ or $n$, the reduction $\bmod p$ ramifies, so that some components get identified. To describe precisely this ramification, we introduce the following polynomials:

Definition 2. For $k \in \mathbb{Z}, k \geq 1$, we define $\Phi_{k}, \Psi_{k} \in \mathbb{Z}[x]$ to be the polynomials determined by the condition:

$$
\Phi_{k}\left(\lambda+\frac{1}{\lambda}\right)= \begin{cases}\frac{\lambda^{k}-1}{\lambda-1} \lambda^{(-k+1) / 2} & k \text { odd } \\ \frac{\lambda^{k}-1}{\lambda^{2}-1} \lambda^{-k / 2+1} & k \text { even }\end{cases}
$$

and

$$
\Psi_{k}\left(\lambda+\frac{1}{\lambda}\right)= \begin{cases}\frac{\lambda^{k}+1}{\lambda+1} \lambda^{(-k+1) / 2} & k \text { odd } \\ \left(\lambda^{k}+1\right) \lambda^{-k / 2} & k \text { even }\end{cases}
$$

for every $\lambda \in \mathbb{C}^{*}$.

Remark 3. These polynomials satisfy the following property: for $A \in \mathrm{SL}_{2}(\mathbb{K})$ with $\mathbb{K}$ an algebraically closed field of characteristic 0 or coprime with $k$ :

- $\Phi_{k}(\operatorname{tr}(A))=0$ if and only if $A^{k}=\mathrm{Id}$ and $A \neq \pm \mathrm{Id}$;

- $\Psi_{k}(\operatorname{tr}(A))=0$ if and only if $A^{k}=-\mathrm{Id}$ and $A \neq-\mathrm{Id}$.

This remark follows easily from viewing $\lambda$ in Definition 2 as an eigenvalue of a matrix with determinant 1 . The following formulae are going to be useful 
later:

$$
\begin{aligned}
\Psi_{2 k}(\operatorname{tr}(A)) & =\operatorname{tr}\left(A^{k}\right) \quad \forall A \in \mathrm{SL}_{2} \\
\Psi_{2 k}(x)-2 & = \begin{cases}(x-2) \Phi_{k}(x)^{2} & k \text { odd } \\
\left(x^{2}-4\right) \Phi_{k}(x)^{2} & k \text { even }\end{cases} \\
\Psi_{2 k}(x)+2 & = \begin{cases}(x+2) \Psi_{k}(x)^{2} & k \text { odd } \\
\Psi_{k}(x)^{2} & k \text { even }\end{cases}
\end{aligned}
$$

The proofs of these formulae and other properties of $\Phi_{k}$ and $\Psi_{k}$ are provided in Appendix C

We describe now $X(T(m, n))$. Using coordinates

$$
\begin{aligned}
& x=\operatorname{tr}_{a} \\
& y=\operatorname{tr}_{b} \\
& z=\operatorname{tr}_{a b^{-1}}
\end{aligned}
$$

we have

$$
X(T(2, n))=X^{a b}(T(2, n)) \cup\left\{(x, y, z) \in \mathbb{C}^{3} \mid x=\Psi_{n}(y)=0\right\},
$$

and, for $m>2$,

$$
\begin{aligned}
X(T(m, n))=X^{a b}(T(m, n)) \cup\left\{(x, y, z) \in \mathbb{C}^{3} \mid\right. & \left.\Phi_{m}(x)=\Phi_{n}(y)=0\right\} \\
& \cup\left\{(x, y, z) \in \mathbb{C}^{3} \mid \Psi_{m}(x)=\Psi_{n}(y)=0\right\}
\end{aligned}
$$

In both cases there are $(m-1)(n-1) / 2+1$ components, including $X^{a b}(T(m, n))$.

The components other than $X^{a b}(T(m, n))$ are lines in the coordinates $(x, y, z)$, as they are defined by $x=x_{0}$ and $y=y_{0}$, for some values $x_{0}, y_{0} \in \mathbb{C}$.

To understand ramifications we use the following lemma, which is straightforward keeping in mind that the map sending each element of a field of characteristic $p$ to its $p$ th power is a morphisms of the field:

Lemma 11. For a prime $p>2$, if $p \mid k$ and $k=p^{r} k^{\prime}$ with $r$ maximal we have:

$$
\Phi_{k}(u) \equiv\left\{\begin{array}{ll}
\Phi_{k^{\prime}}(u)^{p^{r}}(u-2)^{\left(p^{r}-1\right) / 2} & \text { for } k \text { odd, } \\
\Phi_{k^{\prime}}(u)^{p^{r}}\left(u^{2}-4\right)^{\left(p^{r}-1\right) / 2} & \text { for } k \text { even, }
\end{array} \quad \bmod p,\right.
$$

and

$$
\Psi_{k}(u) \equiv\left\{\begin{array}{ll}
\Psi_{k^{\prime}}(u)^{p^{r}}(u+2)^{\left(p^{r}-1\right) / 2} & \text { for } k \text { odd. } \\
\Psi_{k^{\prime}}(u)^{p^{r}} & \text { for } k \text { even, }
\end{array} \quad \bmod p .\right.
$$

In the statement of the lemma, notice that $\Phi_{1}=\Psi_{1}=1$.

Notice that in characteristic $p$ an element has trace \pm 2 precisely when its $p$-th power is equal to $\pm \mathrm{Id}$. In our situation, when $x$ or $y$ is $\pm 2 \bmod p$, the representation can still be irreducible. Thus:

Corollary 12. Let $\mathbb{F}$ be an algebraically closed field of characteristic $p>2$. If $p \mid n$, then the $(m-1)(n-1) / 2$ components of $X(T(m, n))$ containing irreducible characters collapse to $(m-1)\left(n^{\prime}+1\right) / 2$ components in $X(T(m, n))_{\mathbb{F}}$, all of them containing irreducible characters, where $n^{\prime}$ is coprime with $p$ satisfying $n=p^{r} n^{\prime}$. 
More specifically, among the $(m-1)(n-1) / 2$ components present in characteristic zero, $(m-1)\left(n-p^{r}\right) / 2$ collapse to $(m-1)\left(n^{\prime}-1\right) / 2$ components in groups of $p^{r}$, and $(m-1)\left(p^{r}-1\right) / 2$ collapse to $(m-1) / 2$ components (that contain irreducible characters, as $z$ may take arbitrary values).

The components with irreducible characters are pairwise disjoint, but we may ask how they intersect $X^{a b}(T(m, n))$.

Lemma 13. In characteristic 0 or $p$ coprime with $m$ and $n, X^{a b}(T(m, n))$ intersects each other irreducible component of $X(T(m, n))$ transversely in two distinct points.

When reducing mod $p$, if $p \mid n$ then $X^{a b}(T(m, n))$ intersects the components with $y= \pm 2$ tangentially at single point.

Proof. A necessary and sufficient condition for a representation to be abelian or reducible is that the trace of the commutator of the generators $a$ and $b$ is 2; namely, that the coordinates satisfy

$$
x^{2}+y^{2}+z^{2}-x y z-4=0 .
$$

The discriminant in $z$ of this equation is $(x-2)(x+2)(y-2)(y+2)$. This implies that the intersection with a line given by $x=x_{0}$ and $y=y_{0}$ is transverse and consists precisely of two points if and only if $x_{0} \neq \pm 2$ and $y_{0} \neq \pm 2$. This holds in characteristic 0 or coprime with $m$ and $n$.

When $p \mid n$, if $y_{0} \neq \pm 2$ then the discriminant does not vanish and the previous discussion applies. So assume $y_{0}=2$. Notice that the abelianisation $\pi_{1}\left(\mathbf{S}^{3} \backslash T(m, n)\right) \rightarrow \mathbb{Z}$ maps $a$ to $n$ and $b$ to $m$. Hence, for any element $\gamma \in$ $\pi_{1}\left(\mathbf{S}^{3} \backslash T(m, n)\right)$ whose abelianisation generates $\mathbb{Z}$, if $\tau=\operatorname{tr}_{\gamma}$, then by (13) $X^{a b}(T(m, n))$ is parametrised by:

$$
x=\operatorname{tr}_{\gamma^{n}}=\Psi_{2 n}(\tau), \quad y=\operatorname{tr}_{\gamma^{m}}=\Psi_{2 m}(\tau), \quad z=\operatorname{tr}_{\gamma^{-m+n}}=\Psi_{2|m-n|}(\tau) .
$$

We are going to show that the derivatives of $x$ and $y$ with respect to $\tau$ vanish at any intersection point with coordinates $\left(x_{0}, 2, z_{0}\right)$ (this yields tangency with the component defined by $x=x_{0}$ and $y=y_{0}$ ). Since $p \mid n$, by Lemma 11 $x=\Psi_{2 n}(\tau) \cong\left(\Psi_{2 n}(\tau)\right)^{p}$ and its derivative $\bmod p$ with respect to $\tau$ vanishes for any $\tau$. For the derivative of $y$, write $y=2+\left(\Psi_{2 m}(\tau)-2\right)$ and use (14):

$$
y(\tau)=2+ \begin{cases}(\tau-2) \Phi_{m}(\tau)^{2} & \text { for } k \text { odd } \\ \left(\tau^{2}-4\right) \Phi_{m}(\tau)^{2} & \text { for } k \text { even }\end{cases}
$$

As $x_{0} \neq \pm 2 \bmod p$, then $\tau_{0} \neq \pm 2$, hence $\Phi_{m}\left(\tau_{0}\right)=0$ and the derivative of $y$ with respect to $\tau$ at $\tau=\tau_{0}$ vanishes.

When $y=-2$, the proof is similar, using (15).

\section{Satellite knots}

We will study in detail two examples, both with companion the trefoil knot. Recall from the section on torus knots that the fundamental group of the trefoil knot $3_{1}$ is

$$
\pi_{1}\left(\mathbf{S}^{3} \backslash T(2,3)\right) \cong\left\langle\beta, \gamma \mid \beta^{2}=\gamma^{3}\right\rangle,
$$


where the fibre of the Seifert fibration is $\beta^{2}=\gamma^{3}$ and $\gamma^{-1} \beta$ represents a meridian.

From this presentation, the character variety of the trefoil has coordinates $\left(\operatorname{tr}_{\beta}, \operatorname{tr}_{\gamma}, \operatorname{tr}_{\gamma^{-1} \beta}\right)$. It has two components. Both components correspond to characters of representations of quotients of the group: the characters of the first component correspond to representations where the centre is sent to minus the identity

$$
X^{i r r}\left(3_{1}\right)=\left\{\left(\operatorname{tr}_{\beta}, \operatorname{tr}_{\gamma}, \operatorname{tr}_{\gamma^{-1} \beta}\right) \in \mathbb{C}^{3} \mid \operatorname{tr}_{\beta}=0, \operatorname{tr}_{\gamma}=1\right\},
$$

the characters of the second component correspond to abelian representations

$$
X^{a b}\left(3_{1}\right)=\left\{\left(\operatorname{tr}_{\beta}, \operatorname{tr}_{\gamma}, \operatorname{tr}_{\gamma^{-1} \beta}\right) \in \mathbb{C}^{3} \mid \operatorname{tr}_{\beta}=\operatorname{tr}_{\gamma^{-1} \beta}^{3}-3 \operatorname{tr}_{\gamma^{-1} \beta}, \operatorname{tr}_{\gamma}=\operatorname{tr}_{\gamma^{-1} \beta}^{2}-2\right\},
$$

as $\gamma^{-1} \beta$ represents a meridian. Because every representation of the satellite knots we will be considering induces a representation of the trefoil knot, one way to determine the representations, and hence the characters, of the satellite knots is to establish which representations of the patterns (in our case the $T(2,4)$ torus link, and the Whitehead link) can be glued along the common boundary to representations of the trefoil knot to give global representations.

\section{EXAMPLE: A CABLE KNOT $K_{c}$}

This knot exterior is obtained by gluing the exterior of the trefoil knot to the exterior of the $T(2,4)$-torus link in such a way that the fibre of the trefoil knot is identified to the meridian of one of the components of $T(2,4)$ while the meridian of the trefoil knot is glued to the longitude: this ensures that the resulting knot has tunnel number one. A presentation for the torus link is

$$
\pi_{1}\left(\mathbf{S}^{3} \backslash T(2,4)\right) \cong\left\langle\alpha, b \mid b \alpha b^{-1} \alpha=\alpha b^{-1} \alpha b\right\rangle .
$$

Here $\alpha$ and $b$ represent meridians of the two components of $T(2,4)$. One easily observes that the longitude associated to $b$ is $\alpha b^{-1} \alpha b$, so that the exterior of the cable knot has the following presentation:

$$
\pi_{1}\left(\mathbf{S}^{3} \backslash K_{c}\right) \cong\left\langle\alpha, \beta \mid b=\beta^{2}, \gamma^{-1} \beta=\alpha b^{-1} \alpha b, \beta^{2}=\gamma^{3}, b \alpha b^{-1} \alpha=\alpha b^{-1} \alpha b\right\rangle .
$$

The variety of characters of $T(2,4)$ has coordinates $\left(\operatorname{tr}_{\alpha}, \operatorname{tr}_{b}, \operatorname{tr}_{\alpha b^{-1}}\right)$ and two components. One that maps the centre to the identity:

$$
X^{i r r}(T(2,4))=\left\{\left(\operatorname{tr}_{\alpha}, \operatorname{tr}_{b}, \operatorname{tr}_{\alpha b^{-1}}\right) \in \mathbb{C}^{3} \mid \operatorname{tr}_{\alpha b^{-1}}=0\right\}
$$

and the abelian component

$X^{a b}(T(2,4))=\left\{\left(\operatorname{tr}_{\alpha}, \operatorname{tr}_{b}, \operatorname{tr}_{\alpha b^{-1}}\right) \in \mathbb{C}^{3} \mid \operatorname{tr}_{\alpha}^{2}+\operatorname{tr}_{b}^{2}+\operatorname{tr}_{\alpha b^{-1}}^{2}-\operatorname{tr}_{\alpha} \operatorname{tr}_{b} \operatorname{tr}_{\alpha b^{-1}}-4=0\right\}$.

To compute $X\left(K_{c}\right)$ we use coordinates

$$
t=\operatorname{tr}_{\alpha}, z=\operatorname{tr}_{\beta}, x=\operatorname{tr}_{\alpha \beta^{-1}} .
$$

We start with $X\left(3_{1}\right)$ and $X(T(2,4))$ and we add the relations:

$$
\begin{aligned}
& \operatorname{tr}_{b}=\operatorname{tr}_{\beta^{2}}=z^{2}-2 \\
& \operatorname{tr}_{\alpha b^{-1}}=\operatorname{tr}_{\alpha \beta^{-2}}=z x-t \\
& \operatorname{tr}_{\gamma}=\operatorname{tr}_{\beta^{-1} \alpha^{-1} \beta^{2} \alpha^{-1}}=t z^{2} x-t^{2} z-z^{3}-z x^{2}+t x+3 z \\
& \operatorname{tr}_{\beta \gamma^{-1}}=\operatorname{tr}_{\alpha b^{-1} \alpha b}=t z^{3} x-t^{2} z^{2}-z^{4}-z^{2} x^{2}+t^{2}+4 z^{2}-2
\end{aligned}
$$


We assume first that the restriction to $\pi_{1}\left(\mathbf{S}^{3} \backslash 3_{1}\right)$ lies in $X^{i r r}\left(3_{1}\right)$. This imposes the conditions $z=0$ and $\operatorname{tr}_{\gamma}=1$, which in (16) yield

$$
\operatorname{tr}_{b}=-2, \quad \operatorname{tr}_{\alpha b^{-1}}=-t, \quad 1=t x, \quad \operatorname{tr}_{\beta \gamma^{-1}}=t^{2}-2
$$

These equalities are incompatible with $t_{\alpha b^{-1}}=0$, hence the intersection with $X^{i r r}(T(2,4))$ is empty. On the other hand, these equalities imply that

$$
\operatorname{tr}_{\alpha}^{2}+\operatorname{tr}_{b}^{2}+\operatorname{tr}_{\alpha b^{-1}}^{2}-\operatorname{tr}_{\alpha} \operatorname{tr}_{b} \operatorname{tr}_{\alpha b^{-1}}-4=0
$$

that defines $X^{a b}(T(2,4))$. Thus we get a first component

$$
X_{1}\left(K_{c}\right)=\left\{(t, z, x) \in \mathbb{C}^{3} \mid z=0, t x=1\right\} .
$$

Next we suppose that the restriction to $\pi_{1}\left(\mathbf{S}^{3} \backslash 3_{1}\right)$ lies in $X^{a b}\left(3_{1}\right)$, namely it is abelian. Therefore the corresponding representations factor through a Dehn filling on $T(2,4)$. One way to compute the Dehn filling is to add a generator $\eta$ and the relations

$$
\beta=\eta^{3}, \quad \gamma=\eta^{2},
$$

as those are the relations that abelianize the trefoil. The quotient of $\pi_{1}\left(\mathbf{S}^{3} \backslash K_{c}\right)$ by these relations is

$$
\pi_{1}\left(\mathbf{S}^{3} \backslash K_{c}\right) /\left\langle\beta=\eta^{3}, \gamma=\eta^{2}\right\rangle \cong\left\langle\alpha, \eta \mid\left(\alpha^{-1} \eta^{6}\right)^{2}=\eta^{11}\right\rangle .
$$

Thus we get the group of the torus knot $T(2,11)$, and therefore

$$
X\left(K_{c}\right) \cong X_{1}\left(K_{c}\right) \cup X(T(2,11)) .
$$

The six components of $X(T(2,11))$ in the coordinates $(t, z, x)$ become:

$$
X_{2}\left(K_{c}\right)=\left\{(t, z, x) \in \mathbb{C}^{3} \mid t=x z, \quad z^{5}-z^{4}-4 z^{3}+3 z^{2}+3 z-1=0\right\},
$$

for the five curves of irreducible representations, and

$$
X^{a b}\left(K_{c}\right)=\left\{(t, z, x) \in \mathbb{C}^{3} \mid x=t^{5}-5 t^{3}+5 t, z=t^{6}-6 t^{4}+9 t^{2}-2\right\} .
$$

This last component is the abelian one. Notice that the polynomial equation says that $x$ equals the trace of $\alpha^{5}$ and $z$ that of $\alpha^{6}$. Also notice that the five components in $X_{2}\left(K_{c}\right)$ collapse to a single one mod 11, because

$$
\left(z^{5}-z^{4}-4 z^{3}+3 z^{2}+3 z-1\right) \equiv(z-2)^{5} \quad \bmod 11 .
$$

EXAMPle: A WhiteheAd DOUBLE $K_{w}$

In this example, the cable space $S^{3}-T(2,4)$ is replaced with the exterior of the Whitehead link, with presentation

$$
\pi_{1}\left(\mathbf{S}^{3} \backslash 5_{1}^{2}\right)=\left\langle\alpha, b \mid \alpha b^{-1} \alpha^{-1} b \alpha^{-1} b^{-1} \alpha=b^{-1} \alpha b^{-1} \alpha^{-1} b \alpha^{-1} b^{-1} \alpha b\right\rangle,
$$

where $\alpha$ and $b$ are meridians, see Figure 3 . The gluing is as in the previous example, thus giving the following presentation

$$
\begin{gathered}
\pi_{1}\left(\mathbf{S}^{3} \backslash K_{w}\right) \cong\langle\alpha, \beta| b=\beta^{2}, \gamma^{-1} \beta=\alpha b^{-1} \alpha^{-1} b \alpha^{-1} b^{-1} \alpha b, \beta^{2}=\gamma^{3}, \\
\left.\alpha b^{-1} \alpha^{-1} b \alpha^{-1} b^{-1} \alpha=b^{-1} \alpha b^{-1} \alpha^{-1} b \alpha^{-1} b^{-1} \alpha b\right\rangle .
\end{gathered}
$$


Claim 14. There is no character in $X\left(K_{w}\right)$ whose restriction to the trefoil lies in $X^{\text {irr }}\left(3_{1}\right)$.

Proof. Assume there is such a character $\chi_{\rho}$. The gluing requires that a meridian of the Whitehead link, $b$, is glued to the fibre of the trefoil. Hence, $\rho(b)=-\mathrm{Id}$, i.e. it is trivial in $\mathrm{PSL}_{2}(\mathbb{C})$. We notice that the image by $\rho$ of the longitude that commutes with $b$ must also be trivial in $\mathrm{PSL}_{2}(\mathbb{C})$, because each component of the Whitehead link is a trivial knot and the linking number between the two components vanishes. As there is no representation in $X^{\text {irr }}\left(3_{1}\right)$ whose restriction to the peripheral subgroup is trivial in $\mathrm{PSL}_{2}(\mathbb{C})$, we get a contradiction.

Hence all characters in $X\left(K_{w}\right)$ restrict to abelian characters in $X^{a b}\left(3_{1}\right)$. Therefore, we obtain the same representations we get by replacing the trefoil knot exterior by a solid torus. In other terms, these representations correspond to representations of the knot obtained by Dehn-filling one of the two components of the Whitehead link $\left(5_{1}^{2}\right)_{r}$ :

$$
X\left(K_{h}\right) \cong X\left(\left(5_{1}^{2}\right)_{r}\right)
$$

where $r \in \mathbb{Q}$ is the slope of the filling. To determine $r$, we add the generator $\eta$ and the relations

$$
\beta=\eta^{3}, \quad \gamma=\eta^{2},
$$

to the presentation of $\pi_{1}\left(\mathbf{S}^{3} \backslash K_{w}\right)$ as in the previous example, because those are precisely the abelianisation relations of the trefoil. In particular $\eta$ is a longitude that commutes with $b$ (i.e. they are in the same component and in the same choice of peripheral group in the conjugacy class). Thus, we get the presentation

$$
\pi_{1}\left(\mathbf{S}^{3} \backslash\left(5_{1}^{2}\right)_{r}\right)=\left\langle\alpha, b, \gamma \mid b=\gamma^{6}, \gamma=\alpha b^{-1} \alpha^{-1} b \alpha^{-1} b^{-1} \alpha b, b \gamma=\gamma b\right\rangle .
$$

Hence, the filling meridian is $b \gamma^{-6}$, i.e. the filling slope is $r=-1 / 6$.

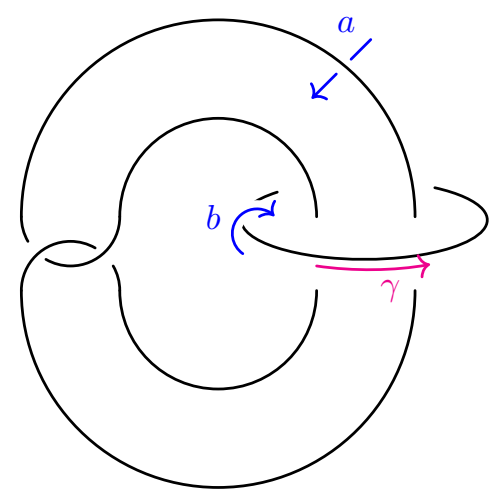

Figure 3: The meridians $a$ and $b$ and the longitude $u$.

To understand the manifold from the Dehn filling with slope $b \gamma^{-6}$, the standard procedure is to cut open along a disc bounded by the component of the link with meridian $b$, apply 6 turns, glue back again, so that the new filling slope becomes $1 / 0=\infty$. This leads to a twist knot with twelve half-twists, that would not be alternate, and simplifies to 11 half-twists as in Figure 4 . This is the two-bridge knot $11 / 23$. The variety of characters of this two-bridge knot has been discussed in Section 4. It ramifies for $p=23$. 

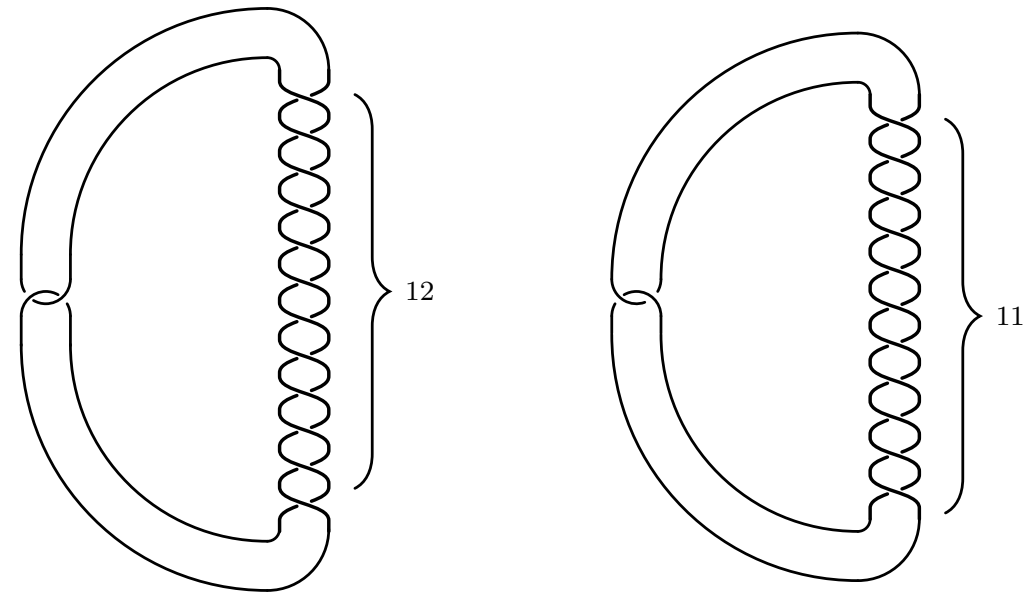

Figure 4: The projection on the left has 12 half-twists but it is not alternate, it simplifies to the projection on the right, which is a diagram of the 2-bridge knot $11 / 23$.

\section{Appendix A Computations for the $8_{5}$}

From Figure 1, using Wirtinger presentation we compute:

$$
\pi_{1}\left(\mathbf{S}^{3} \backslash 8_{5}\right) \cong\left\langle\alpha, \beta, \gamma \mid\left[\alpha^{-1}, \gamma^{-1}\right] \alpha\left[\beta^{-1}, \alpha\right]=\gamma,\left[\beta^{-1}, \alpha\right] \beta[\gamma, \beta]=\gamma\right\rangle
$$

Next we perform elementary changes to obtain a presentation with two generators. Firstly we introduce the elements $w=\alpha \gamma$ and $z=\beta \gamma$ and we eliminate $\alpha$ and $\beta$ :

$$
\begin{aligned}
\pi_{1}\left(\mathbf{S}^{3} \backslash 8_{5}\right) \cong\langle\gamma, w, z| w^{-1} \gamma^{-1} w^{2} z^{-1} w \gamma^{-1} z w^{-1} & \\
& \left.=z^{-2} w \gamma^{-1} z w^{-1} z^{2} \gamma^{-1} z^{-1}=1\right\rangle
\end{aligned}
$$

Setting $a=w^{-1} z^{2}$ and eliminating $w$ we get:

$$
\begin{aligned}
\pi_{1}\left(\mathbf{S}^{3} \backslash 8_{5}\right) \cong\langle\gamma, a, z| a z^{-2} \gamma^{-1} z^{2} a^{-1} z^{2} a^{-1} z\left(a^{-1} \gamma^{-1} z a\right) z^{-2} & = \\
\left.a^{-1} \gamma^{-1} z a \gamma^{-1}=1\right\rangle &
\end{aligned}
$$

The second relation allows to write $z=\gamma a \gamma a^{-1}$ and to simplify the bracket in the first one:

$$
a z^{-2} \gamma^{-1} z^{2} a^{-1} z^{2} a^{-1} z \gamma z^{-2}=1
$$

From this relation we get

$$
a z^{-2} \gamma^{-1} z^{2} a^{-1}=z^{2} \gamma^{-1} z^{-1} a z^{-2},
$$

which tells that $\gamma^{-1} z^{-1} a$ is conjugate to $\gamma^{-1}$. Taking traces it yields the equation

$$
t^{2} x^{2} y+t\left(-x^{3}-2 x y^{2}+2 x-1\right)+x^{2} y+y^{3}-3 y=0
$$

Next taking traces on the relation

$$
\gamma^{-1} z^{2} a^{-1} z^{2} a^{-1} z \gamma=z^{2} a z^{2}
$$


gives

$$
\begin{gathered}
\left(t^{2} x^{3} y^{2}-2 t x^{4} y-2 t x^{2} y^{3}-y^{2} x t^{2}+x^{5}+2 x^{3} y^{2}+x y^{4}+6 t x^{2} y+t y^{3}\right. \\
\left.-t x y-5 x^{3}-5 x y^{2}-2 t y+x^{2}+y^{2}+5 x-2\right) \\
\times\left(t^{3} x^{4} y^{3}-3 t^{2} x^{5} y^{2}-3 t^{2} x^{3} y^{4}-y^{3} x^{2} t^{3}+3 t x^{6} y+6 t x^{4} y^{3}+3 t x^{2} y^{5}\right. \\
+9 t^{2} x^{3} y^{2}+2 t^{2} x y^{4}-x^{7}-3 x^{5} y^{2}-3 x^{3} y^{4}-x y^{6}-15 t x^{4} y \\
-16 t x^{2} y^{3}-t y^{5}-4 y^{2} x t^{2}+7 x^{5}+14 x^{3} y^{2}+7 x y^{4} \\
\left.+18 t x^{2} y+4 t y^{3}-14 x^{3}-14 x y^{2}-3 t y+7 x+1\right)=0
\end{gathered}
$$

We consider the ideal of the polynomials generated by (17) and (18). We then use symbolic software to look for its prime decomposition (namely, the decomposition of its radical) and we get the five components we described. This gives an algebraic set that contains $X\left(8_{5}\right)$. We check that it is precisely $X\left(8_{5}\right)$ exploiting the fact that each component intersects $t=0$ in one of the points described in Section 5

The equations are invariant by the symmetry $(t, x, y) \mapsto(-t, x,-y)$, because $t$ and $y$ are traces of elements whose projections to $\mathbb{Z} / 2 \mathbb{Z}$ are nontrivial, and $x$ is the trace of an element that projects to zero in $\mathbb{Z} / 2 \mathbb{Z}$.

\section{Appendix B Computations for the $8_{18}$}

To obtain a presentation of its fundamental group, we start with three meridians $\alpha, \beta$ and $\gamma$ in Figure 2, We apply the map induced by the period $\phi_{*}^{ \pm 1}$ on these generators, which is equivalent to moving right and left in Figure 5 .
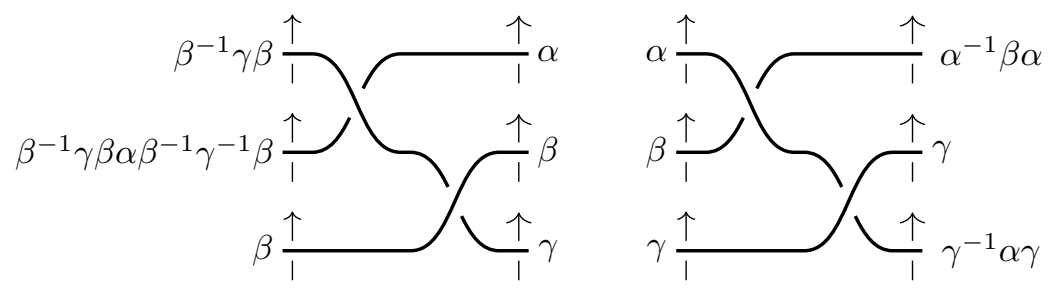

Figure 5: The generators $\alpha, \beta$ and $\gamma$ and their image by $\phi_{*}^{ \pm 1}$

If we iterate this process once in each direction, we obtain the same elements in the fundamental group, yielding to relations. More precisely, the upper and lower strands yield

$$
\begin{aligned}
\beta^{-1} \gamma \beta \alpha^{-1} \beta^{-1} \gamma^{-1} \beta \gamma \beta \alpha \beta^{-1} \gamma^{-1} \beta & =\alpha^{-1} \beta^{-1} \alpha \gamma \alpha^{-1} \beta \alpha \\
\beta^{-1} \gamma \beta \alpha \beta^{-1} \gamma^{-1} \beta & =\gamma \alpha \gamma^{-1} \alpha^{-1} \beta \alpha \gamma \alpha^{-1} \gamma^{-1}
\end{aligned}
$$

Since one relation is redundant, we skip the relation of the middle strand, for it is longer. As they are written, these relations establish that the meridians $\alpha, \beta$ and $\gamma$ are conjugate, so that by taking traces we would get the equality $t=t$. Instead, by modifying them slightly, we derive other equivalent relations, from 
which we compute and equate traces:

$$
\begin{aligned}
\beta \gamma \alpha \gamma^{-1} \alpha^{-1} \beta \alpha \gamma & =\gamma \beta \alpha \beta^{-1} \gamma^{-1} \beta \gamma \alpha \\
\gamma \beta \alpha^{-1} \beta^{-1} \gamma^{-1} \beta \gamma \beta \alpha \beta^{-1} & =\beta \alpha^{-1} \beta^{-1} \alpha \gamma \alpha^{-1} \beta \alpha \beta^{-1} \gamma \\
\gamma^{-1} \beta \gamma \alpha \gamma^{-1} \alpha^{-1} \beta \alpha \gamma & =\beta \alpha \beta^{-1} \gamma^{-1} \beta \gamma \alpha \\
\gamma \alpha \gamma^{-1} \alpha^{-1} \beta \alpha \gamma \alpha^{-1} & =\beta^{-1} \gamma \beta \alpha \beta^{-1} \gamma^{-1} \beta \gamma
\end{aligned}
$$

Taking traces to (19) and writing them as polynomials in our coordinates yields:

$$
\begin{gathered}
-t^{6} z+t^{6}+t^{4} x z+2 t^{4} y z+t^{4} z^{2}-2 t^{4} y-t^{3} w z-2 t^{2} x y z-t^{2} x z^{2} \\
\quad-t^{2} y^{2} z-t^{2} y z^{2}-t^{4}+t^{3} w-t^{2} x^{2}+t^{2} x z+t^{2} y^{2}+t w x z+t w y z+t w z^{2} \\
+x y^{2} z+t^{2} y-2 t^{2} z-t w y-t w z-w^{2} z+x^{2} y+y z^{2}-z^{3}+t^{2}-y+3 z=0
\end{gathered}
$$

We do the same for (20):

$$
\begin{gathered}
t^{6} x+t^{5} w x-t^{4} x^{2} y-t^{6}-t^{5} w+t^{4} w^{2}-t^{4} x^{2}-t^{4} x y-2 t^{4} x z-t^{3} w x^{2}-3 t^{3} w x y \\
-t^{3} w x z+t^{2} x^{3} y+2 t^{2} x^{2} y^{2}+t^{2} x^{2} y z+t^{4} x+2 t^{4} y+t^{4} z+3 t^{3} w x+t^{3} w y+t^{2} w^{2} x-t^{2} w^{2} y \\
-t^{2} x^{2} y+2 t^{2} x^{2} z+2 t^{2} x y z+t^{2} x z^{2}-t w x^{2} y+t w x^{2} z+2 t w x y^{2}-x^{3} y z-x^{2} y^{3}+t^{4} \\
-t^{3} w-3 t^{2} w^{2}-t^{2} x z-t^{2} y^{2}-t^{2} y z+3 t w x y-2 t w x z+2 t w y z-x^{2} y^{2}-x^{2} z^{2} \\
-2 x y^{2} z-2 t^{2} x-t^{2} y-t w x+t w z+x^{2} y-y z^{2}-t^{2}+t w+x z+y+2=0
\end{gathered}
$$

For (21):

$$
\left(t^{2}-y-z\right)\left(t^{3} z-t^{3}-t^{2} w-t x z+x t+t y-z t+w x\right)=0
$$

And for (22):

$$
\begin{array}{r}
-\left(t^{2}-y-z\right)\left(t^{4} z-2 t^{4}-t^{2} x z-t^{2} z^{2}+2 t^{2} x-t w y+t w z+x y^{2}-x y z\right. \\
\left.+x z^{2}+3 t^{2}-t w+y z-2 x-1\right)=0
\end{array}
$$

According to 8 for instance, since the presentation of the group has three generators, there is an additional polynomial relation between the coordinates of the character variety. In our situation, since $t=t r_{\alpha}=t r_{\beta}=t r_{\gamma}$ the equation reads:

$$
w^{2}+\left(t^{3}-t x-t y-t z\right) w-t^{2} x-t^{2} y-t^{2} z+x y z+3 t^{2}+x^{2}+y^{2}+z^{2}-4=0
$$

It turns out that these five polynomials still do not generate the ideal. To see that, we consider again the action of the period $\phi$. The induced action on the characters is given by $\chi \mapsto \chi \circ \phi_{*}^{-1}$, where $\phi_{*}$ is the action on the fundamental group, By Figure 5 (right) $\phi_{*}^{-1}$ is given by

$$
\begin{array}{lll}
\alpha & \quad & \alpha^{-1} \beta \alpha \\
\beta & \mapsto & \gamma \\
\gamma & & \gamma^{-1} \alpha \gamma
\end{array}
$$

We consider the ideal generated by taking the trace in Equations (19), (20), (21) and (22), their image by $\phi_{*}$, and Equation (23), thus a total of 9 polynomials. We then use symbolic software to look for its prime decomposition (namely, the 
decomposition of its radical) and we get the eleven components we described. This gives an algebraic set that contains $X\left(8_{18}\right)$. We check that it is precisely $X\left(8_{18}\right)$ because each component intersects $t=0$ in one of the points described in Section 3 ,

We now describe the action of the involutions $\sigma_{1}$ and $\sigma_{2}$ on the variety of characters.

From Figure 2 the action of $\sigma_{1}$ on the fundamental group is

$$
\begin{array}{lll}
\alpha & & \alpha^{-1} \beta^{-1} \alpha \\
\beta & \mapsto & \alpha^{-1} \beta^{-1} \alpha^{-1} \beta \alpha \\
\gamma & & \alpha^{-1} \beta^{-1} \gamma^{-1} \beta \alpha
\end{array}
$$

Up to inner homomorphisms, it can be simplified as:

$$
\begin{array}{llll}
\alpha & & & \beta^{-1} \\
\beta & \mapsto & \alpha^{-1} \\
\gamma & & \gamma^{-1}
\end{array}
$$

Hence $\sigma_{1}^{*}: X\left(8_{18}\right) \rightarrow X\left(8_{18}\right)$ writes as:

$$
\left(\begin{array}{c}
t \\
x \\
y \\
z \\
w
\end{array}\right) \mapsto\left(\begin{array}{c}
t \\
x \\
t^{2}-z \\
t^{2}-y \\
t^{3}-t y-t z+w
\end{array}\right)
$$

From Figure 2, on the fundamental group $\sigma_{2}$ acts as

$$
\begin{array}{ccc}
\alpha & \gamma \\
\beta \mapsto & \gamma \beta \gamma^{-1} \\
\gamma & \gamma \beta \alpha \beta^{-1} \gamma^{-1}
\end{array}
$$

and on the variety of characters

$$
\left(\begin{array}{c}
t \\
x \\
y \\
z \\
w
\end{array}\right) \mapsto\left(\begin{array}{c}
t \\
y \\
x \\
t^{2}+t w-x y-z \\
w
\end{array}\right)
$$

The map induced by the period $\phi$ on the variety of characters is

$$
\left(\begin{array}{c}
t \\
x \\
y \\
z \\
w
\end{array}\right) \mapsto\left(\begin{array}{c}
t \\
-t^{4}+t^{2} x+t^{2} y+z t^{2}+t^{2}-t w-x z-y \\
t^{2}-z \\
-t^{4} z+t^{4}+t^{2} x z+y z t^{2}+z^{2} t^{2}-t^{2} x-t^{2} y-z w t-x z^{2}+t w-y z+x \\
-t^{5}+t^{3} x+t^{3} y+z t^{3}+2 t^{3}-w t^{2}-x z t-x t-2 t y-z
\end{array}\right)
$$

From this we may derive the description of the action of the symmetry group on the variety of characters given in Section 6 . 


\section{Appendix C The polynomials $\Phi_{k}$ and $\Psi_{k}$}

Here we provide the proof of some of the results stated in Section 7 for the polynomials $\Phi_{k}$ and $\Psi_{k}$ of Definition 2 First of all we rewrite Definition 2 to make computations simpler. The polynomials are determined by the conditions

$$
\Phi_{k}\left(\lambda+\lambda^{-1}\right)= \begin{cases}\frac{\lambda^{k / 2}-\lambda^{-k / 2}}{\lambda^{1 / 2}-\lambda^{-1 / 2}} & k \text { odd } \\ \frac{\lambda^{k / 2}-\lambda^{-k / 2}}{\lambda-\lambda^{-1}} & k \text { even }\end{cases}
$$

and

$$
\Psi_{k}\left(\lambda+\lambda^{-1}\right)= \begin{cases}\frac{\lambda^{k / 2}+\lambda^{-k / 2}}{\lambda^{1 / 2}+\lambda^{-1 / 2}} & k \text { odd } \\ \left(\lambda^{k / 2}+\lambda^{-k / 2}\right) & k \text { even }\end{cases}
$$

for every $\lambda \in \mathbb{C}^{*}$. As $\Psi_{2 k}\left(\lambda+\lambda^{-1}\right)=\lambda^{k}+\lambda^{-k}$, by viewing $\lambda^{ \pm 1}$ as the eigenvalues of a matrix, (13) is clear.

Now we give the proof of (14), but we skip the proof of (15) as it is analogous.

Proof of (14). Set $u=\lambda+\lambda^{-1}$. For every $k$ we have

$$
\Psi_{2 k}(u)-2=\lambda^{k}+\lambda^{-k}-2=\left(\lambda^{k / 2}-\lambda^{-k / 2}\right)^{2}
$$

When $k$ is odd, (24) gives:

$$
\Psi_{2 k}(u)-2=\left(\lambda^{1 / 2}-\lambda^{-1 / 2}\right)^{2}\left(\frac{\lambda^{k / 2}-\lambda^{-k / 2}}{\lambda^{1 / 2}-\lambda^{-1 / 2}}\right)^{2}=(u-2) \Phi_{k}(u)^{2} .
$$

When $k$ is even:

$$
\Psi_{2 k}(u)-2=\left(\lambda-\lambda^{-1}\right)^{2}\left(\frac{\lambda^{k / 2}-\lambda^{-k / 2}}{\lambda-\lambda^{-1}}\right)^{2}=\left(u^{2}-4\right) \Phi_{k}(u)^{2},
$$

which concludes the proof of (14).

Proof of Lemma 11, Let $k=p^{r} k^{\prime}$ be as in the statement of the lemma. The main idea is to use the identity

$$
\lambda^{k / 2} \pm \lambda^{-k / 2} \equiv\left(\lambda^{k^{\prime} / 2} \pm \lambda^{-k^{\prime} / 2}\right)^{p^{r}} \bmod p
$$

and to adapt it to each case. For instance for $k$ odd:

$$
\begin{aligned}
\Phi_{k}(u)=\frac{\lambda^{k / 2}-\lambda^{-k / 2}}{\lambda^{1 / 2}-\lambda^{-1 / 2}} \equiv \frac{\left(\lambda^{k^{\prime} / 2}-\lambda^{-k^{\prime} / 2}\right)^{p^{r}}}{\left(\lambda^{1 / 2}-\lambda^{-1 / 2}\right)^{p^{r}}}\left(\lambda^{1 / 2}-\lambda^{-1 / 2}\right)^{p^{r}-1} & \\
& =\Phi_{k^{\prime}}(u)^{p^{r}}(u-2)^{\left(p^{r}-1\right) / 2} .
\end{aligned}
$$

A similar trick applies to $\Phi_{k}$ for $k$ even and to $\Psi_{k}$ for $k$ either even or odd.

Other properties are $\operatorname{deg}\left(\Phi_{k}\right)=\operatorname{deg}\left(\Psi_{k}\right)=(k-1) / 2$ for $k$ odd, and $\operatorname{deg}\left(\Phi_{k}\right)=$ $k / 2-1$ and $\operatorname{deg}\left(\Psi_{k}\right)=k / 2$ for $k$ even. 
These polynomials are easy to compute if we take into account the recursive relations, as polynomials in $\mathbb{Z}[x]$ :

$$
\Phi_{k}=x \Phi_{k-2}-\Phi_{k-4} \quad \text { and } \quad \Psi_{k}=x \Psi_{k-2}-\Psi_{k-4}
$$

or:

$$
\left\{\begin{array} { l } 
{ \Phi _ { 2 k } = \Phi _ { 2 k - 1 } - \Phi _ { 2 k - 2 } } \\
{ \Phi _ { 2 k + 1 } = ( x + 2 ) \Phi _ { 2 k } - \Phi _ { 2 k - 1 } }
\end{array} \quad \text { and } \quad \left\{\begin{array}{l}
\Psi_{2 k}=(x+2) \Psi_{2 k-1}-\Psi_{2 k-2} \\
\Psi_{2 k+1}=\Psi_{2 k}-\Psi_{2 k-1}
\end{array}\right.\right.
$$

with $\Phi_{1}=\Phi_{2}=\Psi_{1}=1$ and $\Psi_{2}=x$. Here are the polynomials for $k$ up to 10 :

$$
\begin{array}{llrl}
\Phi_{1} & =1 & \Psi_{1} & =1 \\
\Phi_{2} & =1 & \Psi_{2} & =x \\
\Phi_{3} & =x+1 & \Psi_{3} & =x-1 \\
\Phi_{4} & =x & \Psi_{4} & =x^{2}-2 \\
\Phi_{5} & =x^{2}+x-1 & \Psi_{5} & =x^{2}-x-1 \\
\Phi_{6} & =x^{2}-1 & \Psi_{6} & =x^{3}-3 x \\
\Phi_{7} & =x^{3}+x^{2}-2 x-1 & \Psi_{7} & =x^{3}-x^{2}-2 x+1 \\
\Phi_{8} & =x^{3}-2 x & \Psi_{8} & =x^{4}-4 x^{2}+2 \\
\Phi_{9} & =x^{4}+x^{3}-3 x^{2}-2 x+1 & \Psi_{9} & =x^{4}-x^{3}-3 x^{2}+2 x+1 \\
\Phi_{10} & =x^{4}-3 x^{2}+1 & \Psi_{10} & =x^{5}-5 x^{3}+5 x
\end{array}
$$

\section{References}

[1] Michel Boileau and Joan Porti. Geometrization of 3-orbifolds of cyclic type. Astérisque, 272:208, 2001. Appendix A by Michael Heusener and Porti.

[2] S. Boyer and X. Zhang. Finite Dehn surgery on knots. J. Amer. Math. Soc., 9(4):10051050, 1996.

[3] Gerhard Burde and Heiner Zieschang. Knots, volume 5 of De Gruyter Studies in Mathematics. Walter de Gruyter \& Co., Berlin, second edition, 2003.

[4] Michelle Chu. Detecting essential surfaces as intersections in the character variety. Algebr. Geom. Topol., 17(5):2893-2914, 2017.

[5] D. Cooper, M. Culler, H. Gillet, D. D. Long, and P. B. Shalen. Plane curves associated to character varieties of 3-manifolds. Invent. Math., 118(1):47-84, 1994.

[6] Marc Culler and Peter B. Shalen. Varieties of group representations and splittings of 3-manifolds. Ann. of Math. (2), 117(1):109-146, 1983.

[7] Leonard Eugene Dickson. Linear groups: With an exposition of the Galois field theory. with an introduction by W. Magnus. Dover Publications, Inc., New York, 1958.

[8] F. González-Acuña and José María Montesinos-Amilibia. On the character variety of group representations in SL(2, C) and PSL(2, C). Math. Z., 214(4):627-652, 1993.

[9] Michael Heusener, Joan Porti, and Eva Suárez Peiró. Deformations of reducible representations of 3-manifold groups into $\mathrm{SL}_{2}(\mathbf{C})$. J. Reine Angew. Math., 530:191-227, 2001.

[10] Hugh M. Hilden, María Teresa Lozano, and José María Montesinos-Amilibia. On the arithmetic 2-bridge knots and link orbifolds and a new knot invariant. J. Knot Theory Ramifications, 4(1):81-114, 1995.

[11] Hugh M. Hilden, María Teresa Lozano, and José María Montesinos-Amilibia. On volumes and Chern-Simons invariants of geometric 3-manifolds. J. Math. Sci. Univ. Tokyo, 3(3):723-744, 1996. 
[12] Hugh M. Hilden, María Teresa Lozano, and José María Montesinos-Amilibia. On the character variety of periodic knots and links. Math. Proc. Cambridge Philos. Soc., $129(3): 477-490,2000$.

[13] Michael Kapovich. Hyperbolic manifolds and discrete groups, volume 183 of Progress in Mathematics. Birkhäuser Boston Inc., Boston, MA, 2001.

[14] Xiao Song Lin. Representations of knot groups and twisted Alexander polynomials. Acta Math. Sin. (Engl. Ser.), 17(3):361-380, 2001.

[15] Melissa L. Macasieb, Kathleen L. Petersen, and Ronald M. van Luijk. On character varieties of two-bridge knot groups. Proc. Lond. Math. Soc. (3), 103(3):473-507, 2011.

[16] José María Montesinos. Classical tessellations and three-manifolds. Universitext. Springer-Verlag, Berlin, 1987.

[17] Fumikazu Nagasato. Finiteness of a section of the $\operatorname{SL}(2, \mathbb{C})$-character variety of the knot group. Kobe J. Math., 24(2):125-136, 2007.

[18] Tomotada Ohtsuki. Ideal points and incompressible surfaces in two-bridge knot complements. J. Math. Soc. Japan, 46(1):51-87, 1994.

[19] Tomotada Ohtsuki, Robert Riley, and Makoto Sakuma. Epimorphisms between 2-bridge link groups. In The Zieschang Gedenkschrift, volume 14 of Geom. Topol. Monogr., pages 417-450. Geom. Topol. Publ., Coventry, 2008.

[20] Luisa Paoluzzi and Joan Porti. Non-standard components of the character variety for a family of Montesinos knots. Proc. Lond. Math. Soc. (3), 107(3):655-679, 2013.

[21] Luisa Paoluzzi and Joan Porti. Invariant character varieties of hyperbolic knots with symmetries. Math. Proc. Cambridge Philos. Soc., 165(2):193-208, 2018.

[22] Kathleen L. Petersen and Alan W. Reid. Gonality and genus of canonical components of character varieties. arXiv e-prints, page arXiv:1408.3665 Aug 2014.

[23] Joan Porti. Spherical cone structures on 2-bridge knots and links. Kobe J. Math., 21(12):61-70, 2004

[24] Joan Porti and Hartmut Weiss. Deforming Euclidean cone 3-manifolds. Geom. Topol., 11:1507-1538, 2007.

[25] William P. Thurston. The Geometry and Topology of Three-Manifolds. (Electronic version from 2002 available at http://www.msri.org/publications/books/gt3m/), 1980.

[26] Hartmut Weiss. Local rigidity of 3-dimensional cone-manifolds. J. Differential Geom., 71(3):437-506, 2005.

Aix-Marseille Univ, CNRS, Centrale Marseille, I2M, UMR 7373 , 13453 Marseille, France

luisa.paoluzzi@univ-amu.fr

Departament de Matemàtiques, Universitat Autònoma de Barcelona, 08193 Cerdanyola del Vallès, Spain, and BGSMath

porti@mat.uab.cat 